\title{
Mixed Convection in a Two-Sided Lid-Driven Square Cavity Filled with Different Types of Nanoparticles: A Comparative Study Assuming Nanoparticles with Different Shapes
}

\author{
Mostafa Zaydan ${ }^{1}$, Mehdi Riahi ${ }^{1,2, *}$, Fateh Mebarek-Oudina ${ }^{3}$ and Rachid Sehaqui ${ }^{1}$ \\ ${ }^{1}$ Laboratory of Mechanics, Faculty of Sciences Aïn-Chock, University Hassan II, Casablanca, Morocco \\ ${ }^{2}$ Department of Mechanical Engineering, Royal Air School, Marrakech, 40002, Morocco \\ ${ }^{3}$ Department of Physics, Faculty of Sciences, University 20 August 1955, Skikda, 21000, Algeria \\ *Corresponding Author: Mehdi Riahi. Email: mehdi_riahi@hotmail.fr \\ Received: 17 December 2020 Accepted: 01 March 2021
}

\begin{abstract}
Steady, laminar mixed convection inside a lid-driven square cavity filled with nanofluid is investigated numerically. We consider the case where the right and left walls are moving downwards and upwards respectively and maintained at different temperatures while the other two horizontal ones are kept adiabatic and impermeable. The set of nonlinear coupled governing mass, momentum, and energy equations are solved using an extensively validated and a highly accurate finite difference method of fourth-order. Comparisons with previously conducted investigations on special configurations are performed and show an excellent agreement. Meanwhile, attention is focused on the heat transfer enhancement when different nano-particles: $\mathrm{Cu}, \mathrm{Ag}, \mathrm{Al}_{2} \mathrm{O}_{3}, \mathrm{TiO}_{2}$ and $\mathrm{Fe}_{3} \mathrm{O}_{4}$ are incorporated separately in different base fluids such as: Water, Ethylene-glycol, Methanol and Kerosene oil. In this framework, the numerical results related to several mixtures are presented and concern flow pattern and heat transfer curves for various values of Richardson number $[\mathrm{Ri}=0.1,1$ and 10]. It turns out that the choice of the efficient binary mixture for an optimal heat transfer depends not only on the thermophysical properties of the nanofluids but also on the range of the Richardson number. Special attention is devoted to shedding light on the effect of the shape of the nanoparticles on the heat transfer in the case of Water-Ag nanofluid. It is concluded that the spherical shape is more suitable for a better heat transfer enhancement in comparison to the cylindrical ones.
\end{abstract}

\section{KEYWORDS}

Nanofluids; mixed convection; lid-driven square cavity; numerical simulation

\section{List of symbols:}

$\begin{array}{ll}C_{P}: & \text { Specific heat }\left(\mathrm{Jkg}^{-1} \mathrm{~K}^{-1}\right) \\ d_{f}: & \text { Average molecular diameter of the water }(\mathrm{m}) \\ d_{n p}: & \text { Nanoparticles diameter size }(\mathrm{m}) \\ g: & \text { Gravity acceleration }\left(g=9.8 \mathrm{~ms}^{-2}\right) \\ G r: & \text { Grashof number } \\ k: & \text { Thermal conductivity }\left(\mathrm{Wm}^{-1} \mathrm{~K}^{-1}\right) \\ k_{B}: & \text { Boltzmann constant }\left(k_{B}=1.38066 \times 10^{-23} \mathrm{JK}^{-1}\right)\end{array}$




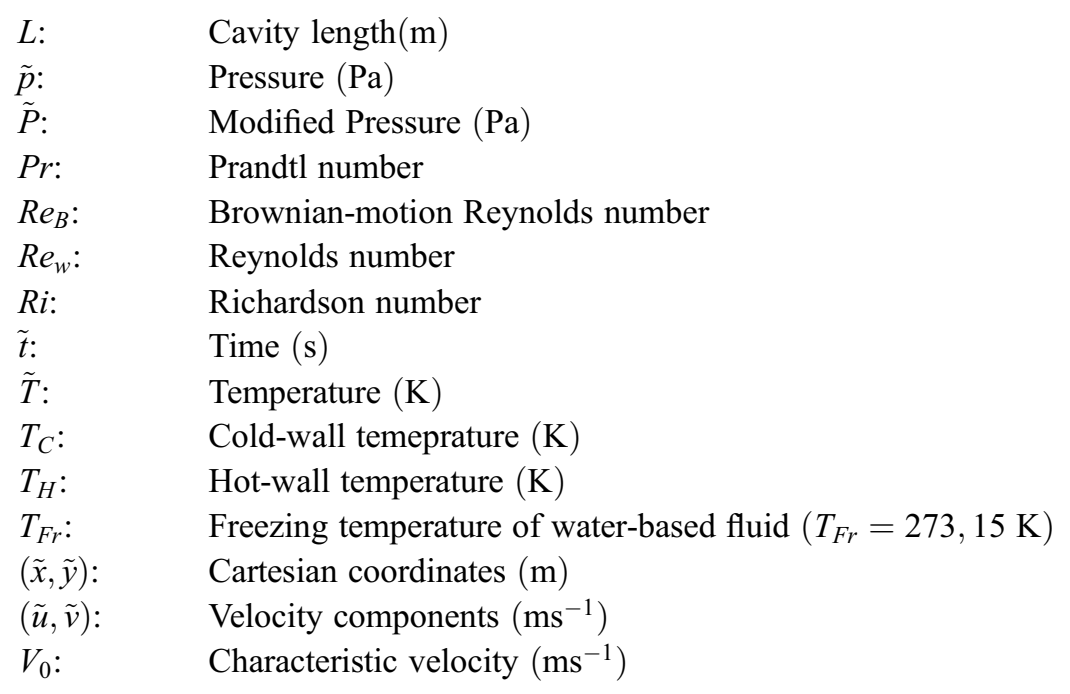

\section{Greek symbols:}

$\begin{array}{ll}\beta: & \text { Thermal expansion coefficient }\left(\mathrm{K}^{-1}\right) \\ \mu: & \text { Dynamic viscosity }(\text { Pas }) \\ \rho: & \text { Density }\left(\mathrm{kg} \mathrm{m}^{-3}\right) \\ \left(\rho C_{P}\right): & \text { Heat capacitance }\left(\mathrm{Jm}^{-3} \mathrm{~K}^{-1}\right) \\ \chi: & \text { Nanoparticles volume fraction } \\ \tilde{G}: & \text { Dimensional variable (e.g., } \tilde{T}) \\ G: & \text { Dimensionless variable (e.g., } T)\end{array}$

$\begin{array}{ll}\text { Subscripts: } & \\ C: & \text { Cold } \\ f: & \text { Fluid } \\ H: & \text { Hot } \\ n f: & \text { Nanofluid } \\ n p: & \text { Nanoparticles } \\ r: & \text { Relative thermophysical properties }\end{array}$

\section{Introduction}

Fluid flow and mixed-convection heat transfer in nanofluid-filled enclosures represent a complicated flow phenomenon due to wall motion that involves forced convection and temperature difference that causes natural convection induced by buoyancy. This kind of flow is of great interest in many industrial sectors, such as the industrial cooling systems applied either for heating or cooling, solar collectors, chemical processing equipment, float glass production, drying technologies, etc. Conventional heat transfer fluids such as water and ethylene glycol, with their low thermal conductivity, limit the performance and compactness of many industrial and engineering electronic devices. Nanofluids are the ideal solution for these equipments since the thermal conductivity of the nanoparticles projected in suspension in the conventional fluid, improves the heat transfer. Different nature of nanoparticles are concerned by this topic including metals $(\mathrm{Al}, \mathrm{Cu})$, oxides $\left(\mathrm{Al}_{2} \mathrm{O}_{3}, \mathrm{TiO}_{2}\right.$, and $\left.\mathrm{CuO}\right)$, carbides $(\mathrm{SiC})$, nitrides (AlN, SiN) or nonmetals (Graphite, carbon nanotubes) while the base fluid is usually a conductive fluid, such as water, ethylene glycol or methanol depending on application field. 
Tiwari et al. [1] investigated numerically the heat transfer enhancement in mixed convection between two-sided lid-driven differentially heated square cavity utilizing nanofluids. The nanofluid used by these authors was Copper-Water nanofluid having Prandlt number $\operatorname{Pr}=6.2$ and solid volume fraction $\chi$ varied up $12 \%$. The upper and the bottom walls are considered thermally insulated while the left and the right moving walls are maintained at different constant temperatures. It was found that the pattern formation and the heat transfer in the cavity can be greatly influenced by both the Richardson number and the direction of the moving walls.

Several studies have been interested by the same purpose in the literatures [2-5]. For instance, Rana et al. [2] have numerically studied the heat transfer enhancement in steady mixed convection flow along a vertical plate with heat source/sink utilizing nanofluids. They emphasized the effect of spherical and cylindrical shaped nanoparticles on the heat transfer using different water-based nanofluids containing $\mathrm{Cu}$, $\mathrm{Ag}, \mathrm{CuO}, \mathrm{Al}_{2} \mathrm{O}_{3}$, and $\mathrm{TiO}_{2}$. It was proved that the use of nanotubes (cylindrical shaped nanoparticles) have higher heat transfer enhancement as compared to spherical shaped nanoparticles. Also, it was shown that $\mathrm{Ag}$ nanoparticles proved to have the highest cooling performance for this vertical plate problem where $\mathrm{TiO}_{2}$ nanoparticles have the lowest. These results have been attributed according to these authors to the high thermal conductivity of $\mathrm{Ag}$ and the low thermal conductivity of $\mathrm{TiO}_{2}$.

In the same framework but in the natural convection, Zaraki et al. [6] have performed a theoretical analysis of boundary layer heat and mass transfer of nanofluids with emphasis on the effects of size, shape and type of nanoparticles, type of base fluid and working temperature. The model adopted in their analysis takes into account the effects of Brownian motion and thermophoresis. It was concluded that the choice of material types of nanoparticles was crucial. Indeed, for some type of nanoparticles significant enhancement of the heat transfer rate was observed, while some others can significantly deteriorate the heat transfer from the surface. For example, the dispersion of $40 \mathrm{~nm}$ spherical zinc-oxide nanoparticles in water enhances the heat transfer while dispersion of $43 \mathrm{~nm}$ of spherical alumina nanoparticles in water leads to a deterioration of the heat transfer [6].

Raza et al. [7] have developed a house code to study the effect of different base fluids on the hydrodynamic and thermal characteristics of Titania nanofluids in cylindrical annulus with discrete heat source. He found that the impact of $\mathrm{TiO}_{2}$ nanofluid heat transfer is related to the basic fluid types.

In this paper, we extend the analysis performed by Tiwari and Das to investigating the heat transfer in this system when different types and shapes of nanoparticles are incorporated in different base fluids. We consider the case where the right wall moves down while the left wall moves to the top which represents the case where the shear and the buoyancy forces act in opposite directions. Using highly validated Fourth-order compact numerical method for the resolution of the nonlinear equations governing the complex physics of the system, we attempt to shed light on the effect of the nature and the shape of the nanoparticles dispersed in different types of base fluids on the heat transfer enhancement in the cavity.

This paper is organized as follows: The studied configuration and its related mathematical formulation are defined in Section 2. Section 3 is devoted to presenting details of the numerical formulation used to solve the heat transfer problem and comparison with the existing results in specific configurations. In Section 4, pertinent results are discussed while the conclusion is addressed in Section 5.

\section{Mathematical Formulation}

The geometry of the present problem is shown in Fig. 1. It consists of a two-dimensional square cavity with the height of $\mathrm{L}$. The temperature of the right wall is considered to be maintained at high temperature of Th as the left sidewall is kept at low temperature of Tc. The horizontal walls are adiabatic and impermeable. The vertical walls are moving with the same velocities in different directions such as the right wall moves downwards with the constant velocity $\mathrm{Vp}$ while the left one moves upwards with $-\mathrm{Vp}$. The cavity is 
filled with a nanofluid with different nanoparticles immersed separately in different base fluids where their pertinent thermophysical properties are given in Tabs. 1 and 2.

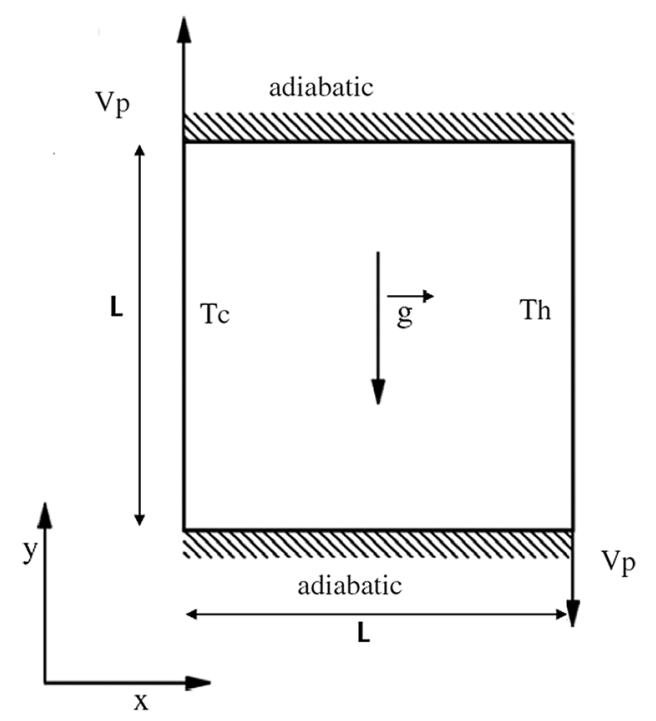

Figure 1: Configuration of the problem in the case of the mixed convection

Table 1: Thermophysical properties of nanoparticles

\begin{tabular}{llllll}
\hline Physical properties & $\mathrm{Al}_{2} \mathrm{O}_{3}$ & $\mathrm{Cu}$ & $\mathrm{TiO}_{2}$ & $\mathrm{Ag}$ & $\mathrm{Fe}_{3} \mathrm{O}_{4}$ \\
\hline$C_{P}(\mathrm{~J} / \mathrm{Kg} \cdot \mathrm{K})$ & 765 & 383 & 686.2 & 235 & 670 \\
$\rho\left(\mathrm{kg} / \mathrm{m}^{3}\right)$ & 3970 & 8933 & 4250 & 10500 & 5180 \\
$\kappa(W / m \cdot K)$ & 40 & 400 & 8.9538 & 429 & 9.7 \\
$\beta\left(K^{-1}\right) \times 10^{5}$ & 0.85 & 1.67 & 0.9 & 1.89 & 0.5 \\
\hline
\end{tabular}

Table 2: Thermophysical properties of base fluids

\begin{tabular}{lllll}
\hline Physical properties & Water & EG & Methanol & Kerosene oil \\
\hline$C_{P}(\mathrm{~J} / \mathrm{Kg} \cdot \mathrm{K})$ & 4179 & 2349 & 2545 & 2090 \\
$\rho\left(\mathrm{kg} / \mathrm{m}^{3}\right)$ & 997.7 & 1132 & 792 & 783 \\
$\kappa(\mathrm{W} / \mathrm{m} \cdot \mathrm{K})$ & 0.613 & 0.258 & 0.2035 & 0.145 \\
$\beta\left(\mathrm{K}^{-1}\right) \times 10^{5}$ & 21 & 57 & 149 & 99 \\
$\operatorname{Pr}$ & 6.2 & 137.48 & 7.38 & 21 \\
\hline
\end{tabular}

The flow is considered incompressible, steady and laminar and governed by the laws of conservation of mass, momentum and energy. Taking into account these assumptions, these equations take the following form:

$\frac{\partial \widetilde{\mathbf{u}}}{\partial \tilde{x}}+\frac{\partial \widetilde{\mathbf{v}}}{\partial \tilde{y}}=0$ 


$$
\begin{aligned}
& \widetilde{\mathrm{u}} \frac{\partial \widetilde{\mathrm{u}}}{\partial \tilde{x}}+\widetilde{\mathrm{v}} \frac{\partial \widetilde{\mathrm{v}}}{\partial \tilde{y}}=-\frac{1}{\rho_{n f, 0}} \frac{\partial \widetilde{\mathrm{P}}}{\partial \tilde{x}}+\frac{\mu_{n f}}{\rho_{n f, 0} \Delta_{2} \widetilde{\mathrm{u}}} \\
& \widetilde{\mathrm{u}} \frac{\partial \widetilde{\mathrm{v}}}{\partial \tilde{x}}+\widetilde{\mathrm{v}} \frac{\partial \widetilde{\mathrm{v}}}{\partial \tilde{y}}=-\frac{1}{\rho_{n f, 0}} \frac{\partial \widetilde{\mathrm{P}}}{\partial \tilde{y}}+\frac{\left[\chi \beta_{s} \rho_{s}+(1-\chi) \rho_{f} \beta_{f}\right]}{\rho_{n f, 0}} g\left(\tilde{T}-\tilde{T}_{0}\right)+\frac{\mu_{n f}}{\rho_{n f, 0}} \Delta_{2} \widetilde{\mathrm{v}} \\
& \tilde{u} \frac{\partial \tilde{T}}{\partial \tilde{x}}+\widetilde{\mathrm{v}} \frac{\partial \tilde{T}}{\partial \tilde{y}}=\alpha_{n f} \Delta_{2} \tilde{T}
\end{aligned}
$$

$\alpha_{n f}$ is the thermal diffusivity of the nanofluid, given by

$\alpha_{n f}=\frac{\kappa_{e f f}}{\left(\rho C_{p}\right)_{n f}}$.

The density $\rho_{n f}$, the heat capacity $\left(\rho C_{p}\right)_{n f}$, and the thermal expansion coefficient $(\rho \beta)_{n f}$, of the nanofluid are obtained from the following equations, respectively:

$\rho_{n f}=\chi \rho_{s}+(1-\chi) \rho_{f}$

$\left(\rho C_{p}\right)_{n f}=\chi\left(\rho C_{p}\right)_{s}+(1-\chi)\left(\rho C_{p}\right)_{f}$

$(\rho \beta)_{n f}=\chi(\rho \beta)_{s}+(1-\chi)(\rho \beta)_{f}$

where $\chi$ being the volume fraction of the solid particles and subscripts $f, n f$ and $s$ designate base fluid, nanofluid and solid nanoparticles, respectively. The effective thermal conductivity of the nanofluid is approximated by the Maxwell-Garnetts model [8] and $\mu_{n f}$ is the effective dynamic viscosity of the nanofluid given respectively by:

$\frac{\kappa_{n f}}{\kappa_{f}}=\frac{\left(\kappa_{s}+2 \kappa_{f}\right)-2 \chi\left(\kappa_{f}-\kappa_{s}\right)}{\left(\kappa_{s}+2 \kappa_{f}\right)+\chi\left(\kappa_{f-} \kappa_{s}\right)}$

$\mu_{n f}=\frac{\mu_{f}}{(1-\chi)^{2.5}}$

In order to proceed to the numerical solution of the system Eqs. (1)-(4) the following dimensionless variables are introduced:

$x=\frac{\tilde{x}}{L}, y=\frac{\tilde{y}}{L}, \mathrm{u}=\frac{\widetilde{\mathrm{u}}}{V_{p}}, \mathrm{v}=\frac{\widetilde{\mathrm{v}}}{V_{p}}, P=\frac{\tilde{P}}{\rho_{n f} V_{p}^{2}}, \mathrm{t}=\frac{\widetilde{\mathrm{t}} \mathrm{V}_{\mathrm{p}}}{\mathrm{L}}, T=\frac{\tilde{T}-T_{C}}{T_{H}-T_{C}}$

By substitution of Eqs. (5) into Eqs. (1)-(4), the following system of equation is derived:

$\frac{\partial \mathrm{u}}{\partial x}+\frac{\partial \mathrm{v}}{\partial y}=0$

$\mathrm{u} \frac{\partial \mathrm{u}}{\partial x}+\mathrm{v} \frac{\partial \mathrm{u}}{\partial y}=-\frac{\partial \mathrm{P}}{\partial x}+\frac{\mu_{n f}}{v_{f} \rho_{n f, 0}} \frac{1}{\operatorname{Re}} \Delta_{2} \mathrm{u}$

$\mathrm{u} \frac{\partial \mathrm{v}}{\partial x}+\mathrm{v} \frac{\partial \mathrm{v}}{\partial y}=-\frac{\partial \mathrm{P}}{\partial y}+\frac{\left[\chi \beta_{s} \rho_{s}+(1-\chi) \rho_{f} \beta_{f}\right]}{\rho_{n f, 0} \beta_{f}} R i . T+\frac{\mu_{n f}}{v_{f} \rho_{n f, 0}} \frac{1}{R e} \Delta_{2} \mathrm{v}$ 
$\mathrm{u} \frac{\partial T}{\partial x}+\mathrm{v} \frac{\partial T}{\partial y}=\frac{\alpha_{n f}}{\alpha_{f}} \frac{1}{P_{r} \cdot \operatorname{Re}} \Delta_{2} T$

The dimensionless parameters appeared in the problem under consideration are:

$\mathrm{Gr}=\frac{\beta_{\mathrm{f}} \mathrm{gH}^{3}\left(\mathrm{~T}_{\mathrm{h}}-\mathrm{T}_{\mathrm{c}}\right)}{\nu_{\mathrm{f}}^{2}}, \quad$ Grash of number

$P_{r}=\frac{v_{f}}{\alpha_{f}}$, Prandtl number

$R e=\frac{V_{p} H}{v_{f}}$, Reynolds number

$R i=\frac{G r}{R e^{2}}$, Richardson number

and the boundary conditions are:

$x=1,0 \leq y \leq 1, u=0, \mathrm{v}=-1, T=1 \quad$ Right wall

$x=0,0 \leq y \leq 1, u=0, \mathrm{v}=1, T=0 \quad$ Left wall

$y=0,0 \leq x \leq 1, u=0, \mathrm{v}=0, \frac{\partial T}{\partial y}=0 \quad$ Bottom wall

$y=1,0 \leq x \leq 1, u=0, \mathrm{v}=0, \frac{\partial T}{\partial y}=0 \quad$ Top wall where

To go from the pressure-velocity formulation $(\mathrm{u}, \mathrm{v}, \mathrm{p})$ to the stream function-vorticity formulation $(\psi, \omega)$

$\mathrm{u}=\frac{\partial \psi}{\partial y}, \mathrm{v}=-\frac{\partial \psi}{\partial x}$ and $\omega=\frac{\partial \mathrm{v}}{\partial \mathrm{x}}-\frac{\partial \mathrm{u}}{\partial \mathrm{y}}$

the system of equations governing the dynamics of the physical problem is written in the following general form

$\frac{\partial^{2} \psi}{\partial x^{2}}+\frac{\partial^{2} \psi}{\partial y^{2}}=-\omega$

$\left(\frac{\partial \psi}{\partial y}\right)\left(\frac{\partial \Gamma}{\partial x}\right)-\left(\frac{\partial \psi}{\partial x}\right)\left(\frac{\partial \Gamma}{\partial y}\right)=\varepsilon\left(\frac{\partial^{2} \Gamma}{\partial \mathrm{x}^{2}}+\frac{\partial^{2} \Gamma}{\partial \mathrm{y}^{2}}\right)+\eta\left(\frac{\partial \mathrm{T}}{\partial \mathrm{x}}\right)$

where

$$
\begin{aligned}
& \Gamma=\omega\left\{\begin{aligned}
\varepsilon & =\frac{\mu_{n f}}{v_{f} \rho_{n f, 0}} \frac{1}{R e} \\
\eta & =\frac{\left[\chi \beta_{s} \rho_{s}+(1-\chi) \rho_{f} \beta_{f}\right]}{\beta_{f} \rho_{n f, 0}} R i
\end{aligned}\right. \\
& \Gamma=T\left\{\begin{array}{l}
\varepsilon=\frac{\alpha_{n f}}{\alpha_{f}} \frac{1}{P_{r} \cdot R e} \\
\eta=0
\end{array}\right.
\end{aligned}
$$

\section{Numerical Method}

For the first and second $\mathrm{x}$-derivatives, the following discretizations with fourth order precision are considered: 
$\frac{\partial \Phi}{\partial x}=\Phi_{x}-\frac{\Delta x^{2}}{6} \frac{\partial^{3} \Phi}{\partial x^{3}}+\mathrm{O}\left(\Delta x^{4}\right)$

$\frac{\partial^{2} \Phi}{\partial x^{2}}=\Phi_{x x}-\frac{\Delta x^{2}}{12} \frac{\partial^{4} \Phi}{\partial x^{4}}+\mathrm{O}\left(\Delta x^{4}\right)$

where $\Phi_{x}$ and $\Phi_{x x}$ are the standard second order centered discretizations in the $x$-direction.

Substituting the expressions (18) and (19) and their corresponding $y$-derivatives into the Eqs. (16) and (17) yield the following form of the kinematic equation:

$\psi_{x x}+\psi_{y y}-\frac{\Delta x^{2}}{12} \frac{\partial^{4} \psi}{\partial x^{4}}-\frac{\Delta y^{2}}{12} \frac{\partial^{4} \psi}{\partial y^{4}}+\mathrm{O}\left(\Delta x^{4}, \Delta y^{4}\right)=-\omega$

while the general conservation equation becomes:

$$
\begin{aligned}
& \varepsilon \Gamma_{x x}+\varepsilon \Gamma_{y y}-\varepsilon \frac{\Delta x^{2}}{12}\left(\frac{\partial^{4} \Gamma}{\partial x^{4}}\right)-\varepsilon \frac{\Delta y^{2}}{12}\left(\frac{\partial^{4} \Gamma}{\partial y^{4}}\right)+\mathrm{O}\left(\Delta x^{4}, \Delta y^{4}\right)=\psi_{y} \Gamma_{x}-\psi_{x} \Gamma_{y}-\frac{\Delta y^{2}}{6} \Gamma_{x}\left(\frac{\partial^{3} \psi}{\partial y^{3}}\right) \\
& -\frac{\Delta x^{2}}{6} \psi_{y}\left(\frac{\partial^{3} \Gamma}{\partial x^{3}}\right)+\frac{\Delta x^{2}}{6} \Gamma_{y}\left(\frac{\partial^{3} \psi}{\partial x^{3}}\right)+\frac{\Delta y^{2}}{6} \psi_{x}\left(\frac{\partial^{3} \Gamma}{\partial y^{3}}\right)-\eta T_{x}+\eta \frac{\Delta x^{2}}{6}\left(\frac{\partial^{3} T}{\partial x^{3}}\right)+O\left(\Delta x^{4}, \Delta x^{2}, \Delta y^{2}, \Delta y^{4}\right) .
\end{aligned}
$$

Considering the obtained form of the Eq. (20), one can remark that the fourth $x$ and $y$-derivatives of the stream function should be replaced by their expressions resulting from Eq. (16) where

$\frac{\partial^{4} \psi}{\partial x^{4}}=-\frac{\partial^{2} \omega}{\partial x^{2}}-\frac{\partial^{4} \psi}{\partial x^{2} \partial y^{2}}$

$\frac{\partial^{4} \psi}{\partial y^{4}}=-\frac{\partial^{2} \omega}{\partial y^{2}}-\frac{\partial^{4} \psi}{\partial y^{2} \partial x^{2}}$

And by using the standard form of second-order centered discretization, the Eqs. (22) and (23) can be written as:

$\frac{\partial^{4} \psi}{\partial x^{4}}=-\omega_{x x}-\psi_{x x y y}+\mathrm{O}\left(\Delta x^{2}, \Delta y^{2}\right)$

$\frac{\partial^{4} \psi}{\partial y^{4}}=-\omega_{y y}-\psi_{x x y y}+\mathrm{O}\left(\Delta x^{2}, \Delta y^{2}\right)$

Taking into account these expressions, we obtain finally the following finite difference form of the kinematic equation:

$\Psi_{x x}+\Psi_{y y}=-\omega-\frac{\Delta x^{2}}{12} \omega_{x x}-\frac{\Delta y^{2}}{12} \omega_{y y}-\left(\frac{\Delta x^{2}}{12}+\frac{\Delta y^{2}}{12}\right) \psi_{x x y y}+\mathrm{O}\left(\Delta x^{4}, \Delta x^{2}, \Delta y^{2} \Delta y^{4}\right)$

whose solution is a solution of the stream function Eq. (16) with a fourth-order spatial precision.

The same approach is applied to obtain the fourth-order approximation of the general conservation equations along the $(O x)$ and $(O y)$ axes. For instance, the third and the fourth $x$-derivative are written as:

$\frac{\partial^{3} \Gamma}{\partial x^{3}}=\frac{1}{\varepsilon} \frac{\partial^{2} \psi}{\partial x \partial y} \frac{\partial \Gamma}{\partial x}+\frac{1}{\varepsilon} \frac{\partial \psi}{\partial y} \frac{\partial^{2} \Gamma}{\partial x^{2}}-\frac{1}{\varepsilon} \frac{\partial^{2} \psi}{\partial x^{2}} \frac{\partial \Gamma}{\partial y}-\frac{1}{\varepsilon} \frac{\partial \psi}{\partial x} \frac{\partial^{2} \Gamma}{\partial x \partial y}-\frac{\eta}{\varepsilon} \frac{\partial^{2} \mathrm{~T}}{\partial x^{2}}-\frac{\partial^{3} \Gamma}{\partial x \partial y^{2}}$ 


$$
\begin{aligned}
\frac{\partial^{4} \Gamma}{\partial x^{4}}= & \frac{1}{\varepsilon} \frac{\partial^{3} \psi}{\partial x^{2} \partial y} \frac{\partial \Gamma}{\partial x}+\frac{1}{\varepsilon} \frac{\partial^{2} \psi}{\partial x \partial y} \frac{\partial^{2} \Gamma}{\partial x^{2}}+\frac{1}{\varepsilon} \frac{\partial^{2} \psi}{\partial x \partial y} \frac{\partial^{2} \Gamma}{\partial x^{2}}+\frac{1}{\varepsilon} \frac{\partial \psi}{\partial y} \frac{\partial^{3} \Gamma}{\partial x^{3}}-\frac{1}{\varepsilon} \frac{\partial^{3} \psi}{\partial x^{3}} \frac{\partial \Gamma}{\partial y}-\frac{1}{\varepsilon} \frac{\partial^{2} \psi}{\partial x^{2}} \frac{\partial^{2} \Gamma}{\partial x \partial y} \\
& -\frac{1}{\varepsilon} \frac{\partial^{2} \psi}{\partial x^{2}} \frac{\partial^{2} \Gamma}{\partial x \partial y}-\frac{1}{\varepsilon} \frac{\partial \psi}{\partial x} \frac{\partial^{3} \Gamma}{\partial x^{2} \partial y}-\frac{\eta}{\varepsilon} \frac{\partial^{3} \mathrm{~T}}{\partial x^{3}}-\frac{\partial^{4} \Gamma}{\partial x^{2} \partial \mathrm{y}^{2}}
\end{aligned}
$$

The final form of our fourth order compact formulation (FOCF) scheme of the equations governing the dynamics of the system is as follows:

$\psi_{x x}+\psi_{y y}=-\omega+\mathrm{A}$

$\varepsilon(1+B) \Gamma_{x x}+\varepsilon(1+C) \Gamma_{y y}=\left(\psi_{y}+D\right) \Gamma_{x}-\left(\psi_{x}+E\right) \Gamma_{y}-\frac{\eta}{\Upsilon_{1}} T_{x}+F$

with:

$$
\begin{aligned}
A= & -\frac{\Delta x^{2}}{12} \omega_{x x}-\frac{\Delta y^{2}}{12} \omega_{y y}-\left(\frac{\Delta x^{2}}{12}+\frac{\Delta y^{2}}{12}\right) \Psi_{x x y y}, \quad B=-\frac{1}{\varepsilon} \frac{\Delta x^{2}}{6} \Psi_{x y}+\frac{1}{\varepsilon^{2}} \frac{\Delta x^{2}}{12} \Psi_{y} \Psi_{y} \\
C= & \frac{1}{\varepsilon} \frac{\Delta y^{2}}{6} \Psi_{x y}+\frac{1}{\varepsilon^{2}} \frac{\Delta y^{2}}{12} \Psi_{x} \Psi_{x} \\
D= & \left(\frac{\Delta x^{2}}{12}+\frac{\Delta y^{2}}{12}\right) \Psi_{x x y}-\frac{1}{\varepsilon} \frac{\Delta x^{2}}{12} \Psi_{y} \Psi_{x y}+\frac{1}{\varepsilon} \frac{\Delta y^{2}}{12} \Psi_{x} \Psi_{y y} \\
E= & \left(\frac{\Delta x^{2}}{12}+\frac{\Delta y^{2}}{12}\right) \Psi_{x y y}-\frac{1}{\varepsilon} \frac{\Delta x^{2}}{12} \Psi_{y} \Psi_{x x}+\frac{1}{\varepsilon} \frac{\Delta y^{2}}{12} \Psi_{x} \Psi_{x y} \\
F= & \left(\frac{\Delta x^{2}}{12}+\frac{\Delta y^{2}}{12}\right) \psi_{y} \Gamma_{x y y}-\left(\frac{\Delta x^{2}}{12}+\frac{\Delta y^{2}}{12}\right) \psi_{x} \Gamma_{x x y}-\frac{1}{\varepsilon} \frac{\Delta x^{2}}{6} \psi_{x x} \Gamma_{x y}+\frac{\Delta y^{2}}{6} \psi_{y y} \Gamma_{x y}+\frac{1}{\varepsilon}\left(\frac{\Delta x^{2}}{12}+\frac{\Delta y^{2}}{12}\right) \psi_{x} \psi_{y} \Gamma_{x y} \\
& -\frac{1}{\varepsilon}\left(\frac{\Delta x^{2}}{12}-\frac{\Delta y^{2}}{12}\right) \Gamma_{x} \Gamma_{y}-\left(\frac{\Delta x^{2}}{12}+\frac{\Delta y^{2}}{12}\right) \Gamma_{x x y y}+\eta \cdot G
\end{aligned}
$$

where

$$
\begin{aligned}
G= & \frac{\Upsilon_{2}}{\Upsilon_{1}} \frac{\Delta x^{2}}{12} \psi_{x y} T_{x}+\left(\frac{\Upsilon_{2}}{\Upsilon_{1}}+\frac{1}{\Upsilon_{1}^{2}}\right) \frac{\Delta x^{2}}{12} \psi_{y} T_{x x}-\frac{\Upsilon_{2}}{\Upsilon_{1}} \frac{\Delta x^{2}}{12} \psi_{x x} T_{y}-\left(\frac{\Upsilon_{2}}{\Upsilon_{1}} \frac{\Delta x^{2}}{12}+\frac{1}{\Upsilon_{1}^{2}} \frac{\Delta y^{2}}{12}\right) \psi_{x} T_{x y} \\
& -\frac{1}{\Upsilon_{1}}\left(\frac{\Delta x^{2}}{12}+\frac{\Delta y^{2}}{12}\right) T_{x y y}
\end{aligned}
$$

And

$\Upsilon_{1}=\frac{\mu_{n f}}{v_{f} \rho_{n f, 0}} \frac{1}{R e} ; \Upsilon_{2}=\frac{\alpha_{n f}}{\alpha_{f}} \frac{1}{P_{r} \cdot \operatorname{Re}}$

Note that if A, B, C, D, E and F vanishes, the Eqs. (29) and (30) are reduced to the second order formulation. These equations associated to the boundary conditions are solved numerically by the implicit scheme with alternating directions (ADI: Alternating directions Implicit) method established by Peaceman et al. [9] in 1955 which consists in advancing half a step of time following $x$ and then the second half step following $y$.

Such iterative numerical algorithm requires pseudo time derivatives to be assigned to the equations presenting the physical system such as: 
$\frac{\partial^{2} \psi}{\partial x^{2}}+\frac{\partial^{2} \psi}{\partial y^{2}}=-\omega+\frac{\partial \psi}{\partial \mathrm{t}}$

$\frac{\partial \Gamma}{\partial t}+\left(\frac{\partial \psi}{\partial y}\right)\left(\frac{\partial \Gamma}{\partial x}\right)-\left(\frac{\partial \psi}{\partial x}\right)\left(\frac{\partial \Gamma}{\partial y}\right)=\varepsilon\left(\frac{\partial^{2} \Gamma}{\partial \mathrm{x}^{2}}+\frac{\partial^{2} \Gamma}{\partial \mathrm{y}^{2}}\right)+\eta\left(\frac{\partial \mathrm{T}}{\partial \mathrm{x}}\right)$

The solution of this system will not depend on the time derivative introduced by the numerical scheme since we are dealing with a steady flow configuration where the source of unsteadiness does not exist. Consequently, a first order $(\Delta t)$ accurate which is an implicit Euler step is added to these pseudo time derivatives as well as to the non-linear terms in the general conservation equation. In this framework, the system of Eqs. (32) and (33) is rewritten as follows:

$\psi^{\mathrm{n}}+\Delta \mathrm{t} \frac{\partial^{2} \psi^{\mathrm{n}+1}}{\partial x^{2}}+\Delta \mathrm{t} \frac{\partial^{2} \psi^{\mathrm{n}+1}}{\partial y^{2}}=-\Delta \mathrm{t} \omega^{\mathrm{n}}+\psi^{\mathrm{n}+1}$

$\Gamma^{\mathrm{n}+1}+\Delta t\left(\frac{\partial \psi^{\mathrm{n}}}{\partial y}\right)\left(\frac{\partial \Gamma^{\mathrm{n}+1}}{\partial x}\right)-\Delta t\left(\frac{\partial \psi^{\mathrm{n}}}{\partial x}\right)\left(\frac{\partial \Gamma^{\mathrm{n}+1}}{\partial y}\right)=\varepsilon \Delta \mathrm{t}\left(\frac{\partial^{2} \Gamma^{\mathrm{n}+1}}{\partial x^{2}}+\frac{\partial^{2} \Gamma^{\mathrm{n}+1}}{\partial y^{2}}\right)+\eta \Delta \mathrm{t}\left(\frac{\partial \mathrm{T}^{\mathrm{n}+1}}{\partial x}\right)+\Gamma^{\mathrm{n}}$

This approach has been used recently by Erturk et al. [10] where the solution of the Eqs. (34) and (35) converge always toward that of the steady Eqs. (16) and (17). For avoiding any kind of repetition in this paper, we will not present the different steps used by these authors and the reader should be referred to [10] for more details.

In order to check the convergence of the obtained results during the iterations, several residuals factors are calculated. We define the first residual expression related to the stream function and the general conservation function respectively as follows:

$R E S 1_{\psi}=\max \left(\left|\frac{\psi_{i-1, j}^{n+1}-2 \psi_{i, j}^{n+1}+\psi_{i+1, j}^{n+1}}{\Delta x^{2}}+\frac{\psi_{i, j-1}^{n+1}-2 \psi_{i, j}^{n+1}+\psi_{i, j+1}^{n+1}}{\Delta y^{2}}+\omega_{i, j}^{n+1}-A_{i, j}^{n+1}\right|\right)$

$\operatorname{RES} 1_{\Gamma}=\max \left(\left|\begin{array}{c}\varepsilon\left(1+\mathrm{B}_{i, j}^{n+1}\right)\left(\frac{\Gamma_{i-1, j}^{n+1}-2 \Gamma_{i, j}^{n+1}+\Gamma_{i+1, j}^{n+1}}{\Delta x^{2}}\right)+ \\ \varepsilon\left(1+\mathrm{C}_{i, j}^{n+1}\right)\left(\frac{\Gamma_{i, j-1}^{n+1}-2 \Gamma_{i, j}^{n+1}+\Gamma_{i, j+1}^{n+1}}{\Delta y^{2}}\right)- \\ \left(\frac{\psi_{i, j+1}^{n+1}-\psi_{i, j-1}^{n+1}}{2 \Delta y}+\mathrm{D}_{i, j}^{n+1}\right)\left(\frac{\Gamma_{i+1, j}^{n+1}-\Gamma_{i-1, j}^{n+1}}{2 \Delta x}\right)+ \\ \left(\frac{\psi_{i+1, j}^{n+1}-\psi_{i-1, j}^{n+1}}{2 \Delta x}+\mathrm{E}_{i, j}^{n+1}\right)\left(\frac{\Gamma_{i, j+1}^{n+1}-\Gamma_{i, j-1}^{n+1}}{2 \Delta y}\right)+ \\ \left(\frac{\eta}{\Upsilon_{1}} \frac{T_{i+1, j}^{n+1}-T_{i-1, j}^{n+1}}{2 \Delta x}-F_{i, j}^{n+1}\right)\end{array}\right|\right)$

The value of RES1 is an indication related to the time derivatives introduced by the numerical procedure. It would generally vanish since the solution converges to the steady state.

A second residual parameter, RES2, is defined by the following expressions presenting the difference in absolute value between two successive iteration steps for the variables stream function, vorticity and energy:

$R E S 2_{\psi}=\max \left(\left|\psi_{i, j}^{n+1}-\psi_{i, j}^{n}\right|\right)$ 
$\operatorname{RES} 2_{\Gamma}=\max \left(\left|\Gamma_{i, j}^{n+1}-\Gamma_{i, j}^{n}\right|\right)$

RES2 gives an indication of the significant digit on which the code is iterated.

The third residual parameter, RES3, is similar to RES2 except that it is normalized by the representative value of the previous time step. This makes possible to get an indication later on the maximum percentage variation of change in $\psi$ and $\Gamma$ at each iteration step. RES3 is defined as follows:

$R E S 3_{\psi}=\max \left(\left|\frac{\psi_{i, j}^{n+1}-\psi_{i, j}^{n}}{\psi_{i, j}^{n+1}}\right|\right)$

$R E S 3_{\Gamma}=\max \left(\left|\frac{\Gamma_{i, j}^{n+1}-\Gamma_{i, j}^{n}}{\Gamma_{i, j}^{n+1}}\right|\right)$

In our calculations and for all Rayleigh numbers, we consider that convergence is obtained when both $\operatorname{RES}_{\psi} \leq 10^{-6}$ and $\operatorname{RES} 1_{\Gamma} \leq 10^{-6}$ are reached. This value is chosen to ensure an accurate precision of the solution. At these convergence levels, the second residual parameters are of the order of RES $2_{\psi} \leq 10^{-10}$ and $\operatorname{RES} 2_{\Gamma} \leq 10^{-11}$ which means that the stream function, vorticity and the energy equations are accurate to the precision of 9 and 10 digits respectively after the decimal point, to a grid point and even more accurate to the rest of the meshes. Similar convergence criteria is adopted for the other residuals where RES $3_{\psi} \leq 10^{-10}$ and $\mathrm{RES}_{\Gamma} \leq 10^{-9}$. These small residues guarantee that our solutions are indeed very precise.

\subsection{Boundary Condition for the Vorticity}

Dimensionless boundary conditions for $\psi$ are:

$\psi=0$ for $\mathrm{X}=0, \mathrm{X}=1$ and $0 \leq \mathrm{Y} \leq 1$

$\psi=0$ for $\mathrm{Y}=0, \mathrm{Y}=1$ and $0 \leq \mathrm{X} \leq 1$

For vorticity, Störtkuh et al. [11] have presented an analytical asymptotic solution near the corners of cavity and using finite element bilinear shape functions and they also have presented a singularityremoved boundary condition for vorticity at the corner points as well as at the wall points.

In this study, we follow Störtkuh et al. [11] for the boundary conditions and use the following expressions for calculating vorticity values at the walls:

$\frac{1}{3 \Delta h^{2}}\left[\begin{array}{ccc}\cdot & \cdot & . \\ \frac{1}{2} & -4 & \frac{1}{2} \\ 1 & 1 & 1\end{array}\right] \psi+\frac{1}{9}\left[\begin{array}{ccc}\cdot & \cdot & . \\ \frac{1}{2} & 2 & \frac{1}{2} \\ \frac{1}{4} & 1 & \frac{1}{4}\end{array}\right] \omega=-\frac{V}{\Delta h}$
$\frac{1}{3 \Delta h^{2}}\left[\begin{array}{ccc}\cdot & \cdot & \cdot \\ \cdot & -2 & \frac{1}{2} \\ \cdot & \frac{1}{2} & 1\end{array}\right] \psi+\frac{1}{9}\left[\begin{array}{ccc}\cdot & \cdot & . \\ \cdot & 1 & \frac{1}{2} \\ \cdot & \frac{1}{2} & \frac{1}{4}\end{array}\right] \omega=-\frac{V}{2 \Delta h}$

and at the corner points:

where $\mathrm{V}$ is the velocity of the walls. In our case, this velocity equals 1 for the left vertical wall, -1 for the right vertical wall whereas 0 for the other horizontal walls.

In explicit notation, for the wall points shown in Fig. 2a, the vorticity is calculated as following: 
$\omega_{b}=-\frac{3}{2 \Delta h^{2}}\left(\psi_{d}+\psi_{e}+\psi_{f}\right)-\frac{1}{8}\left(2 \omega_{a}+2 \omega_{c}+\omega_{d}+4 \omega_{e}+\omega_{f}\right)$
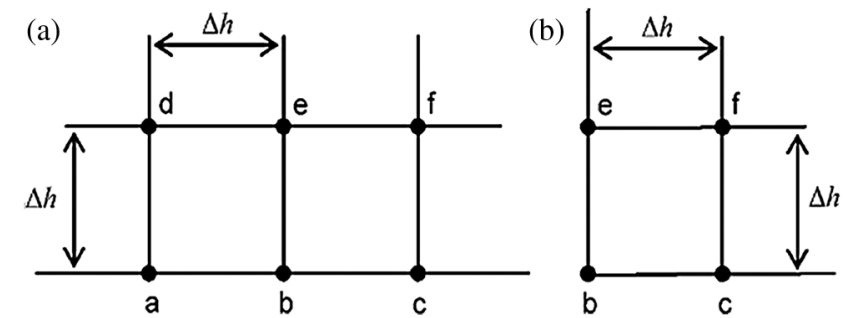

Figure 2: Grid points at the walls and at the corners: (a) wall points and (b) corner points

Similarly, for the corner points shown in Fig. 2b, the vorticity is calculated as the following:

$\omega_{b}=-\frac{3}{2 \Delta h^{2}} \psi_{f}-\frac{1}{4}\left(2 \omega_{c}+2 \omega_{e}+\omega_{f}\right)$

The reader should be referred to Störtkuh et al. [11] for more details on the boundary conditions.

$N u_{\text {local }}=\frac{h_{n f} L}{k_{f}}=-\left.\left(\frac{k_{n f}}{k_{f}}\right) \frac{\partial \theta}{\partial Y}\right|_{Y=0}$

$\overline{N u}=\int_{0}^{1}\left(-\left.\left(\frac{k_{n f}}{k_{f}}\right) \frac{\partial \theta}{\partial Y}\right|_{Y=0}\right) d x$

The averaged and the local heat transfer rates at the left cold wall of the cavity are presented in terms of the averaged Nusselt numbers, $\overline{N u}$ and the local Nusselt number $N u_{\text {local }}$ defined respectively as follows.

\subsection{Code Validation}

A good concordance is found by comparing the results obtained by our numerical code with those of de Vahl Davis [12], Markatos et al. [13], Hadjisophcleous et al. [14], Tiwari et al. [1], El Bouihi et al. [15], Khanafer et al. [16], Barakos et al. [17], Fusegi et al. [18] and Goodarzi et al. [19]. This comparison is performed on the problem of natural convection in a differentially heated square enclosure filled with air having the Prandlt number $\operatorname{Pr}=0.71$. In Tab. 3, we report the numerical values of the average Nusselt number obtained for different values of the Rayleigh number.

For giving more reliability to our code, we attempt to compare our numerical results with previous works concerned by mixed convection in differentially heated cavity containing air where the top wall is moving with a constant velocity and kept hot while the bottom wall is cooled. The two vertical walls are insulated. At $\mathrm{Gr}=\llbracket 10 \rrbracket \wedge 2$ and for different values of the Reynolds number the obtained results are summarized in Tab. 4. As one can notice, excellent agreement is found by comparing the results obtained by Tiwari et al. [1], Sharif [20], Waheed [21], Khanafer et al. [22], Iwatsu et al. [23] and Cheng [24].

\section{Results and Discussion}

\subsection{Grid Test}

Computations with reasonable accuracy require an optimum choose of the mesh. For that, we start by shedding light on the influence of the size and nodes distribution on the average Nusselt number $\overline{N u}$ and the stream function $\left|\Psi_{\min }\right|$. We report in Tab. 5 results related to these physical concepts for an assigned value of the Richardson number $\mathrm{Ri}=10^{2}$ and the volume fraction $\chi=10 \%$ with a uniform distribution of nodes. As 
one can notice, $\overline{N u}$ and $\left|\Psi_{\min }\right|$ become independent to the number of nodes when the grid $104 \times 104$ is reached. Consequently, this latter mesh size will be adopted in the rest of our calculations.

Table 3: Average Nusselt number comparison with previous works

\begin{tabular}{lllll}
\hline & $R a=10^{3}$ & $R a=10^{4}$ & $R a=10^{5}$ & $R a=10^{6}$ \\
\hline De Vahl Davis [12], FDM & 1.118 & 2.243 & 4.519 & 8.799 \\
Markatos et al. [13], FDM & 1.108 & 2.201 & 4.430 & 8.754 \\
Hadjisophcleous et al. [14] & 1.141 & 2.290 & 4.964 & 10.390 \\
Tiwari et al. [1] FVM & 1.087 & 2.195 & 4.450 & 8.803 \\
El Bouihi et al. [15] ADI second ordre & 1.042 & 2.024 & 4.520 & 8.978 \\
Khanafer et al. [16], FVM & 1.118 & 2.245 & 4.522 & 8.826 \\
Barakos et al. [17], FVM & 1.114 & 2.245 & 4.510 & 8.806 \\
Fusegi et al. [18], 3-D FDM & 1.105 & 2.302 & 4.646 & 9.012 \\
Goodarzi et al. [19], LBM & 1.108 & 2.210 & 4.456 & 8.756 \\
Present work FOCF & 1.117 & 2.242 & 4.922 & 9.917 \\
Difference with [14] & $2.10 \%$ & $2.88 \%$ & $0.84 \%$ & $4.55 \%$ \\
\hline
\end{tabular}

Table 4: Comparison of the Nusselt number with previous works (C.V.M)

\begin{tabular}{llllllll}
\hline Re & $\begin{array}{l}\text { Our } \\
\text { results }\end{array}$ & $\begin{array}{l}\text { Tiwari et al. } \\
{[1]}\end{array}$ & $\begin{array}{l}\text { Sharif } \\
{[20]}\end{array}$ & $\begin{array}{l}\text { Waheed } \\
{[21]}\end{array}$ & $\begin{array}{l}\text { Khanafer et al. } \\
{[22]}\end{array}$ & $\begin{array}{l}\text { Sharif } \\
{[20]}\end{array}$ & $\begin{array}{l}\text { Waheed } \\
{[21]}\end{array}$ \\
\hline 100 & 2.052 & 2.10 & - & 2.0311 & 2.01 & 1.94 & - \\
400 & 4.083 & 3.85 & 4.05 & 4.0246 & 3.91 & 3.84 & 4.14 \\
1000 & 6.599 & 6.33 & 6.55 & 6.4842 & 6.33 & 6.33 & 6.73 \\
\hline
\end{tabular}

Table 5: Values of the physical quantities $\overline{N u}$ and $\left|\Psi_{\min }\right|$ for different grid values

\begin{tabular}{lllllllll}
\hline Grid & $34 \times 34$ & $44 \times 44$ & $64 \times 64$ & $84 \times 84$ & $104 \times 104$ & $124 \times 124$ & $144 \times 144$ & $164 \times 164$ \\
\hline$\overline{N u}$ & 6.29275 & 6.37389 & 6.36743 & 6.31079 & 6.25236 & 6.2512 & 6.251 & 6.2521 \\
$\left|\Psi_{\min }\right|$ & 0.1064 & 0.1064 & 0.1063 & 0.1063 & 0.1062 & 0.1062 & 0.1062 & 0.1062 \\
\hline
\end{tabular}

This grid test is performed on the framework of a mixed convection configuration using square cavity filled with air $(\operatorname{Pr}=0.71)$ with a vertical thermal gradient and moving upper horizontal wall.

\subsection{Heat Transfer and Pattern Formation in Ag-Water Nanofluid}

Our attention is focused on the mixed convective regimes where both forced and natural convections coexist corresponding to a Richardson number range $0.1 \leq \mathrm{Ri} \leq 10$. Typically, the natural convection is negligible, compared to the forced one, when $\mathrm{Ri}<0.1$ whereas it becomes predominant when $\mathrm{Ri}>10$. However, when $\mathrm{Ri}=0.1$ we are in presence of mixed convection regime since both the Rayleigh and the Reynolds numbers are involved in the system. In this case, the forced convection is slightly more 
important in comparison to the natural one, which is also present, since $R a<R e^{2}\left(R a=0.1 R e^{2}\right)$. The mixed convective regime is also considered when $\mathrm{Ri}=10$ but in this case the natural convection is more important in comparison to the forced one since $R a>R e^{2}\left(R a=10 R e^{2}\right)$. The pure forced convection is reached when $\mathrm{Ri}=0(R a=0)$ while the pure natural one is reached when the Richardson number tends to infinity $(R e=0)$.

The configuration we considered in this study is when the left vertical wall moves constantly upward while the right one moves downwards with the same velocity. Here, the buoyancy and shear forces are opposing each other. The Grashof number is fixed at $\mathrm{Gr}=10^{4}$ and variation of the Richadson number is produced by a variation of the Reynolds number.

Fig. 3 illustrates the streamlines (left part) and the isotherms (right part) for Water and Water-Ag nanofluid when gradually increasing the volume fraction $(\chi=0.04,0.2)$ when $\mathrm{Ri}=0.1$. As one can see from the Figs. $3 a-3 c$ and $3 \mathrm{e}-3 \mathrm{~g}$, the flow pattern is not influenced by the presence of the nanoparticles where only one convective roll is formed in the whole cavity. The direction of rotation of this cell is clockwise as it can be deduced from the vertical velocity profile related to this case plotted at the midplane $\left(x=\frac{1}{2}\right)$ in Fig. 6a. This latter exhibits a symmetry point located at the center of the cavity, where

$u\left(x=\frac{1}{2}, y\right)=-u\left(x=\frac{1}{2}, 1-y\right) \quad \forall y \in[0,1]$.

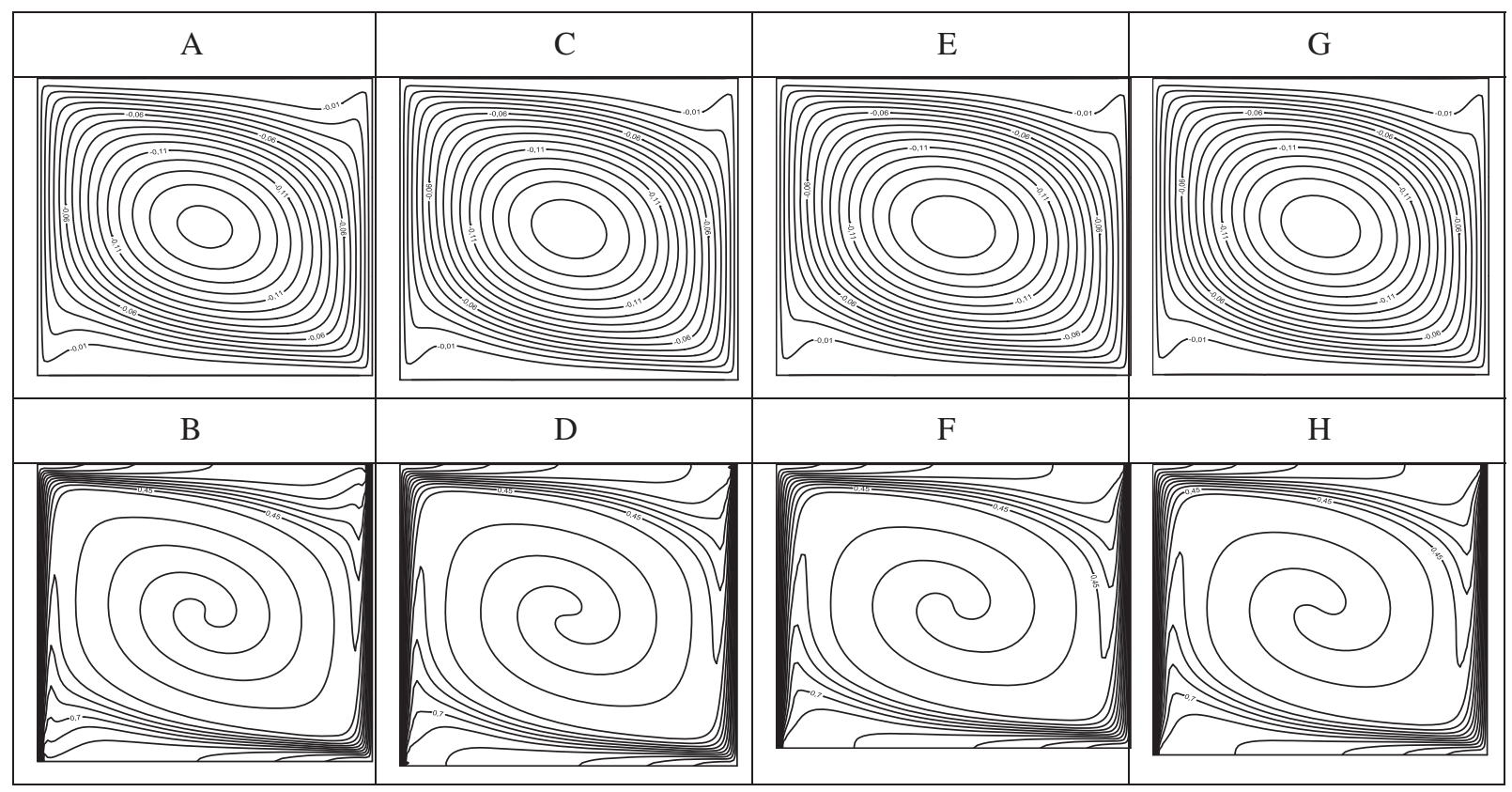

Figure 3: Streamlines (A, C, E, G) and isotherms (B, D, F, H) for Ri $=0.1$ : (A) $\chi=0 \%$; (B) $\chi=0 \%$; (C) $\chi=8 \%$; (D) $\chi=8 \%$; (E) $\chi=16 \%$; (F) $\chi=16 \%$; (G) $\chi=20 \%$; (H) $\chi=20 \%$

This symmetry property means that the flow, at this mid-plan, changes its direction from the upper half of the cavity $\frac{1}{2} \leq y \leq 0$ to the lower one $0 \leq y \leq \frac{1}{2}$. It is to be noted, in addition, that the rotation's direction of the cell affects considerably the fluid flow in the cavity especially in the corners. Here, indeed, only the leftupper and right-lower corners are occupied by the fluid in contrast to the other corners.

The isotherms related to this configuration are depicted in Figs. $3 b-3 d$ and $3 \mathrm{f}-3 \mathrm{~h}$ where a strong convection is noticed for all the values of the nanoparticle fractions. Similarly to the streamlines, the 
isotherms are present in the left-upper and right-lower corners ensuring heat transfer in these regions. The temperature at the horizontal mid-plane $\left(y=\frac{1}{2}\right)$ is constant inside the cavity except near the vertical boundaries due to the presence of two thermal boundary layers near the vertical walls as shown in the Fig. 7a. The temperature function also exhibits the centre of the cavity $\left(x=\frac{1}{2}, y=\frac{1}{2}\right)$ as a symmetry point and it verifies the following law:

$T\left(x, y=\frac{1}{2}\right)=1-T\left(1-x, y=\frac{1}{2}\right) \forall x \in[0,1] /\left[x_{1}, x_{2}\right]$.

The interval $\left[x_{1}, x_{2}\right]$ is a part of the cavity where the temperature is constant having the value $T=0.5$, an average value between the temperatures of the vertical walls. In this Richardson number, $x_{1} \approx 0.15$ and $x_{2}=0.85$.

Decreasing the Reynolds number until $\mathrm{Ri}=1$ is reached, the flow pattern and the heat transfer characteristics are changed as shown in Fig. 4. Indeed, secondary flow composed by three cells is observed for $\chi \leq 8 \%$ : two cells near the left and the right walls rotating in clockwise direction and a main cell placed at the middle of the cavity rotating in the anti-clockwise direction, see Figs. $4 \mathrm{a}-4 \mathrm{c}$ and $4 \mathrm{e}-4 \mathrm{~g}$. In addition, an increase in the nanoparticles concentration beyond $\chi=8 \%$ leads to a stabilization of the flow in the sense that it reduces the three cells into one cell rotating in clockwise direction. This feature is responsible of the appearance of phenomenon commonly known as flow reversal observed in several flow configurations as the Couette-Taylor system with modulated rotation of the cylinders [25-28]. Indeed, it concerns a change in the direction of the vortices and results in discontinuity in the first derivative of the critical Taylor number as well as a jump in critical wave number. This phenomenon can be clearly deduced from the velocity profile plotted at the vertical mid-plan $\left(x=\frac{1}{2}\right)$ in Fig. $7 b$. Meanwhile, we recover a dynamics similar to that where $\mathrm{Ri}<1$, a configuration in which the forced convection is predominant than the natural one, and therefore, one can conclude that an increase in the nanoparticle amount leads to a change in the convection mechanism in the sense that the secondary flow becomes mainly due to forced convection. Moreover, the symmetry property given by the expression (50) for $\mathrm{Ri}=0.1$ remains also valid for $\mathrm{Ri}=1$.

A

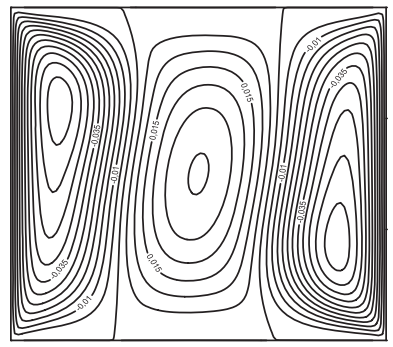

B

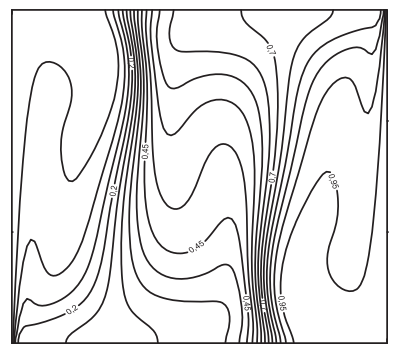

$\mathrm{C}$

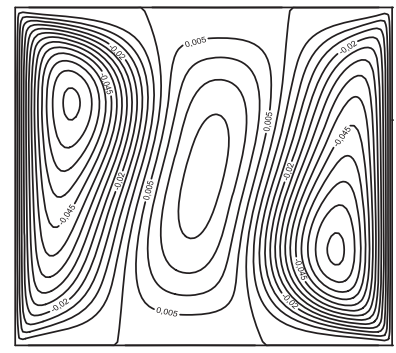

$\mathrm{D}$

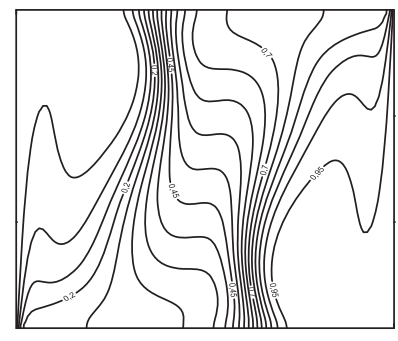

$\mathrm{E}$

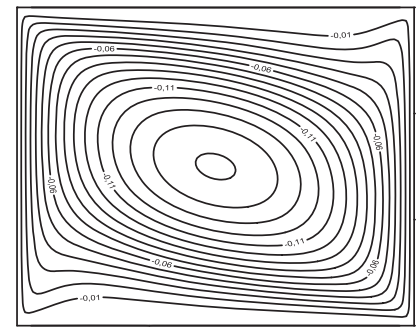

$\mathrm{F}$

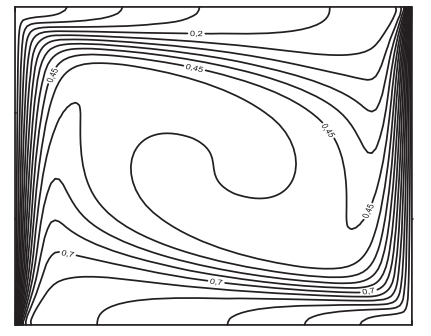

G

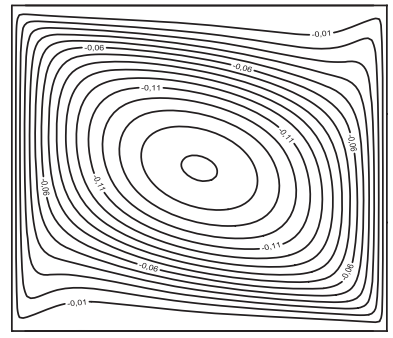

$\mathrm{H}$

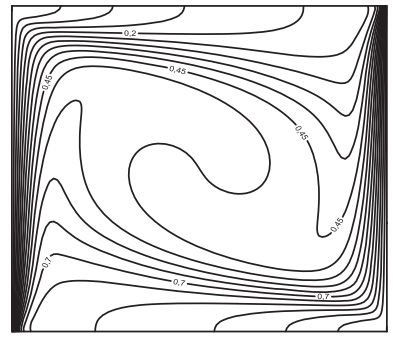

Figure 4: Streamlines (A, C, E, G) and isotherms (B, D, F, H) for Ri $=1$ : (A) $\chi=0 \%$; (B) $\chi=0 \%$; (C) $\chi=8 \%$; (D) $\chi=8 \%$; (E) $\chi=16 \%$; (F) $\chi=16 \%$; (G) $\chi=20 \%$; (H) $\chi=20 \%$ 
Regarding the isotherms, two thermal boundary layers are formed when $\chi \leq 8 \%$ at the middle of the cavity exactly at the junction of the two adjacent vortices discussed above from the streamlines curves, see Figs. 4b-4d and 4f-4h. These boundary layers move towards the vertical walls by increasing the nanoparticles concentration due to the disappearance of the central cell in the streamlines. At the horizontal mid-plane $\left(y=\frac{1}{2}\right)$ the temperature profile related to this case is displayed in Fig. $7 \mathrm{~b}$. As one can see, the nanoparticles volume fraction affects the shape of the temperature profile. Indeed, for $\chi \leq 8 \%$ the temperature has the same characteristic as the case $\mathrm{Ri}=0.1$ except a shrink observed in the region of the cavity where $T=0.5$ defined by the interval $\left[x_{1}, x_{2}\right]$. In this case, the values $x_{1}$ and $x_{2}$ are $x_{1} \approx 0.25$ and $x_{2} \approx 0.65$. This region is suppressed, however, in the case $\chi>8 \%$, where the temperature increases nonlinearly from $T=0$ to $T=1$ at $x=0$ and $x=1$ respectively. Consequently, its symmetry property becomes

$T\left(x, y=\frac{1}{2}\right)=1-T\left(1-x, y=\frac{1}{2}\right) \quad \forall x \in[0,1]$.

The case where natural convection becomes much more important than forced convection corresponds to $\mathrm{Ri}=10$. Here, as it was mentioned by Tiwari et al. [1] it is expected that the nanoparticles do not affect neither the fluid dynamics nor the heat transfer since their brownian motion is not taken into account in the model. This feature can be clearly seen from Fig. 5 related to the streamlines and the isotherms. In fact, the flow structure is composed by three cells similarly to the case where the natural and the forced convections are with the same order of magnitude, $\mathrm{Ri}=1$, with weak amount of nanoparticles. One can also observe the same velocity profiles at the mid-plane $\left(x=\frac{1}{2}\right)$ plotted in Fig. 6c. The nanoparticles do not affect these velocities except a slight increase observed at their maximums by increasing the volume fraction $\chi$. The symmetry described by the expression (50) is also verified for this case, $\mathrm{Ri}=10$. The temperature inside the cavity at $y=\frac{1}{2}$ increases generally in the $x$ direction and exhibits an inflection point at the cavity center $\left(x=\frac{1}{2}, y=\frac{1}{2}\right)$ giving rise to the appearance of two symmetric extrema in the neighborhood of the cavity. Here, a slight decrease of the temperature is observed, see Fig. 7c. The symmetry law given by the expression (52) describes also the temperature profile in this case.

A

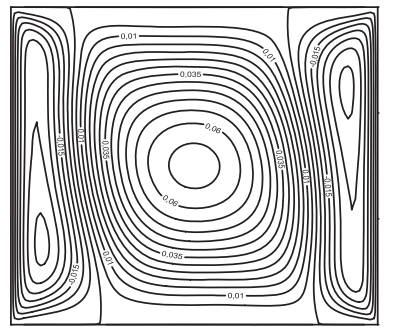

B

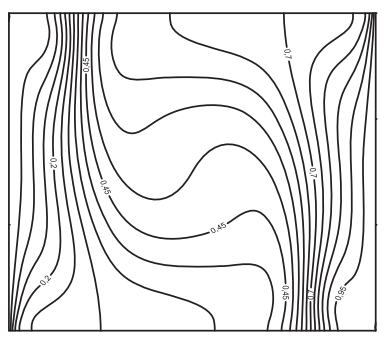

C

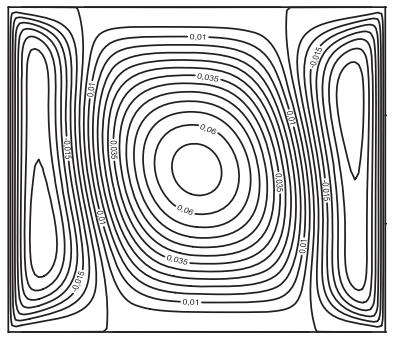

$\mathrm{D}$

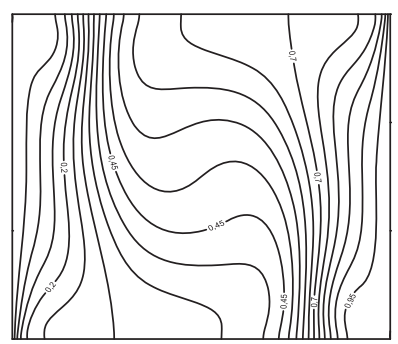

$\mathrm{E}$

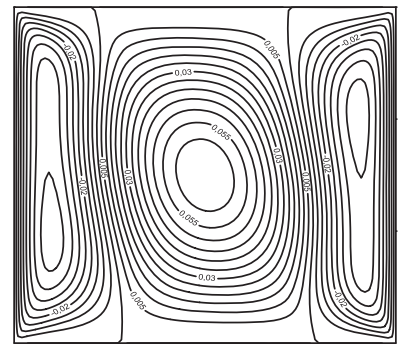

$\mathrm{F}$

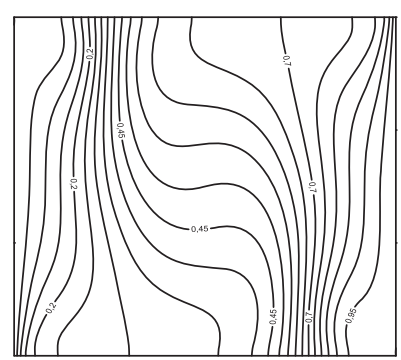

G

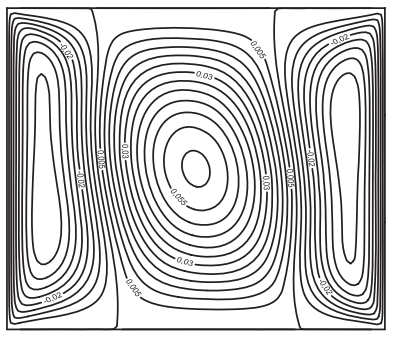

$\mathrm{H}$

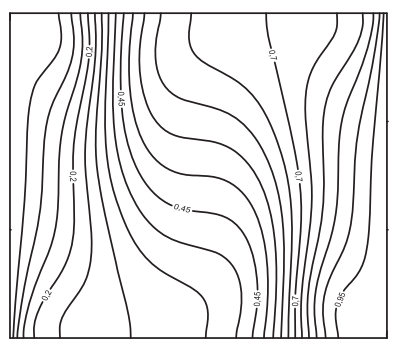

Figure 5: Streamlines (A, C, E, G) and isotherms (B, D, F, H) for Ri $=10$ : (A) $\chi=0 \%$; (B) $\chi=0 \%$; (C) $\chi=8 \%$; (D) $\chi=8 \%$; (E) $\chi=16 \%$; (F) $\chi=16 \%$; (G) $\chi=20 \%$; (H) $\chi=20 \%$ 
(a)

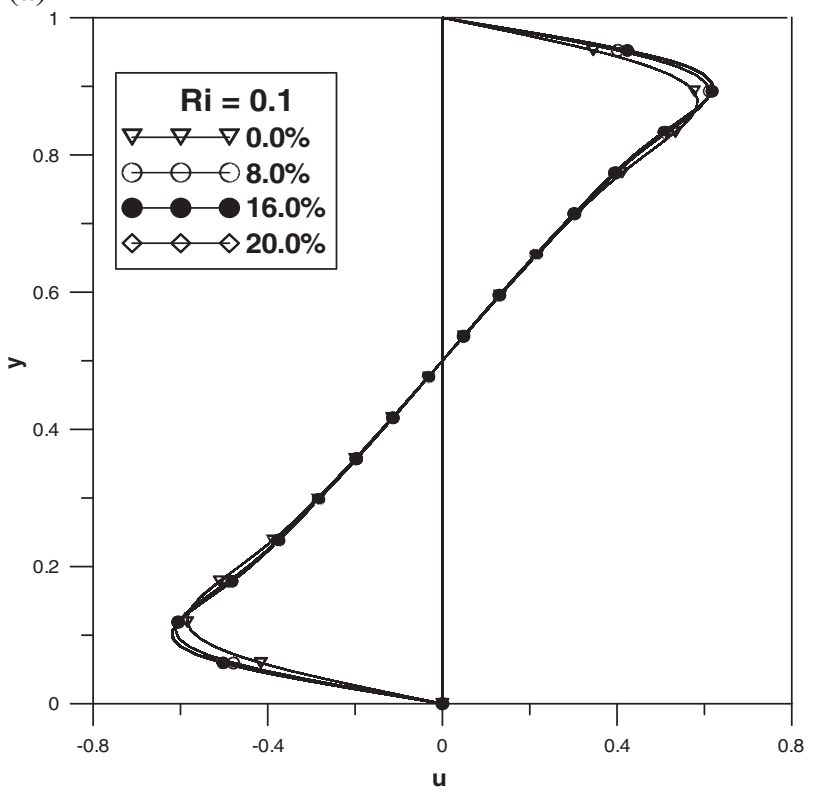

(b)

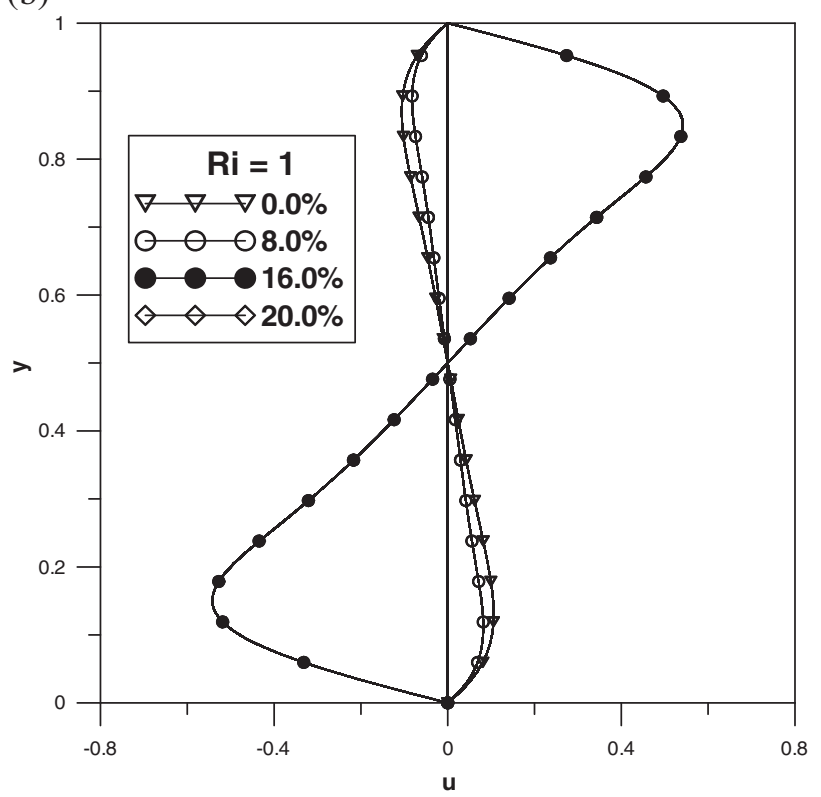

(c)

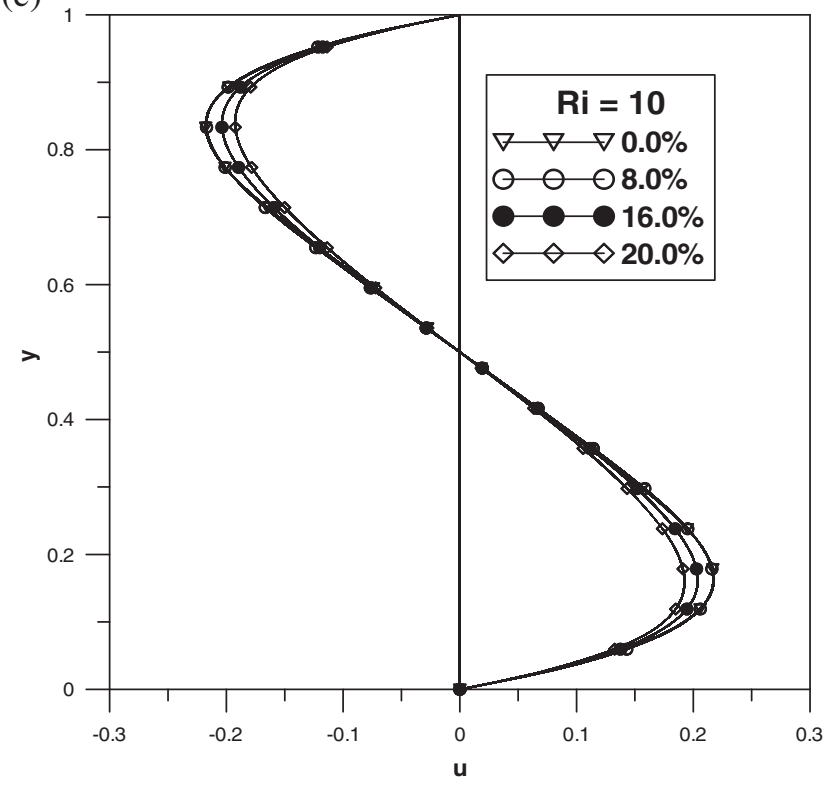

Figure 6: Vertical mid-plane u-velocity: (a) $\mathrm{Ri}=0.1$; (b) $\mathrm{Ri}=1$; (c) $\mathrm{Ri}=10$

We will now discuss the heat transfer in our system by referring to the local Nusselt number $\mathrm{Nu}$ at the cold wall located at the left side of the cavity. Fig. 8 displays the evolution of $\mathrm{Nu}$ for different values of $\chi$ and $\mathrm{Ri}$. It is apparent from the Fig. 8 that the Nusselt number reaches a maximum value at the lower part of the wall, $(y=0)$. One can expect a maximal value of Nusselt number at the upper part of the wall, $(y=1)$ if the left and the right walls are moving respectively downwards and upwards as it was turned out in Tiwari et al. [1]. One can also notice a decrease in $\mathrm{Nu}$ versus the coordinatey. This behavior is independent to the Richardson number and the nanoparticles volume fraction. However, for $\mathrm{Ri}=0.1$ and $\mathrm{Ri}=1$ the effect of the nanoparticles volume fraction is observed only in the lower part of 
the wall where the parameter $\chi$ enhances the heat transfer by increasing the Nusselt number. It is to be noticed that a great jump in $\mathrm{Nu}$ number is observed at $\mathrm{Ri}=1$ by increasing the amount of the nanoparticles where $\mathrm{Nu}=23$ when $\chi \leq 8 \%$ and $\mathrm{Nu}=92$ when $\chi>8 \%$ in contrast to the case $\mathrm{Ri}=10$ where the volume fraction $\chi$ weakly enhances the heat transfer along the wall except near the boundaries $y=0$ and $y=1$.

(a)

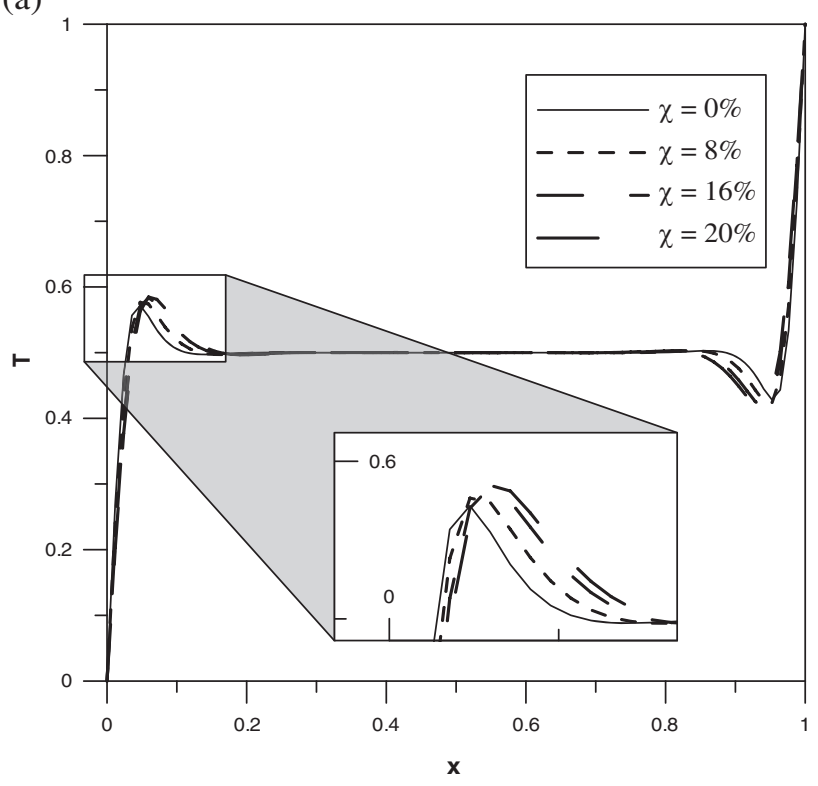

(b)

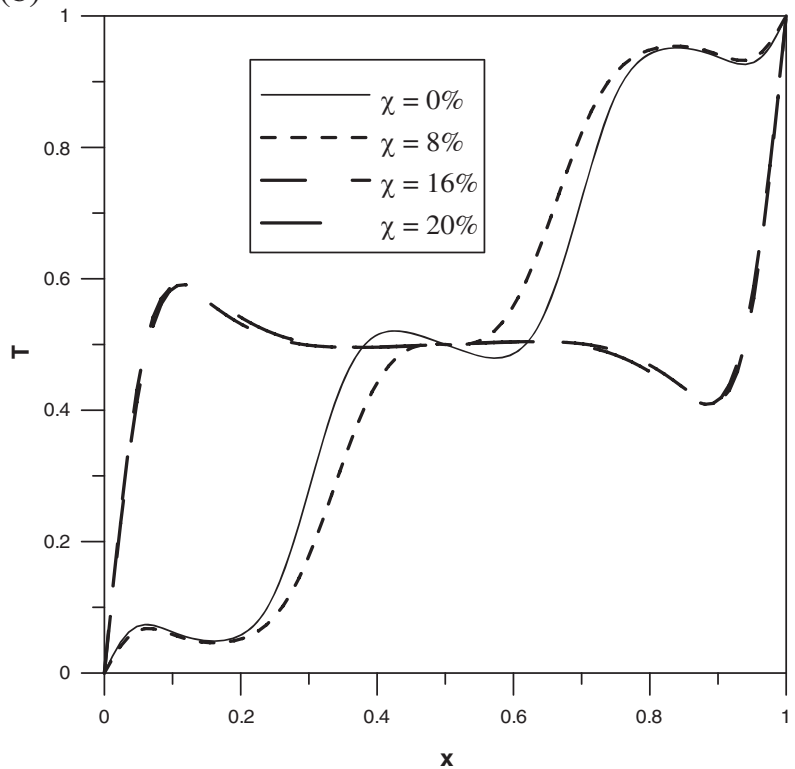

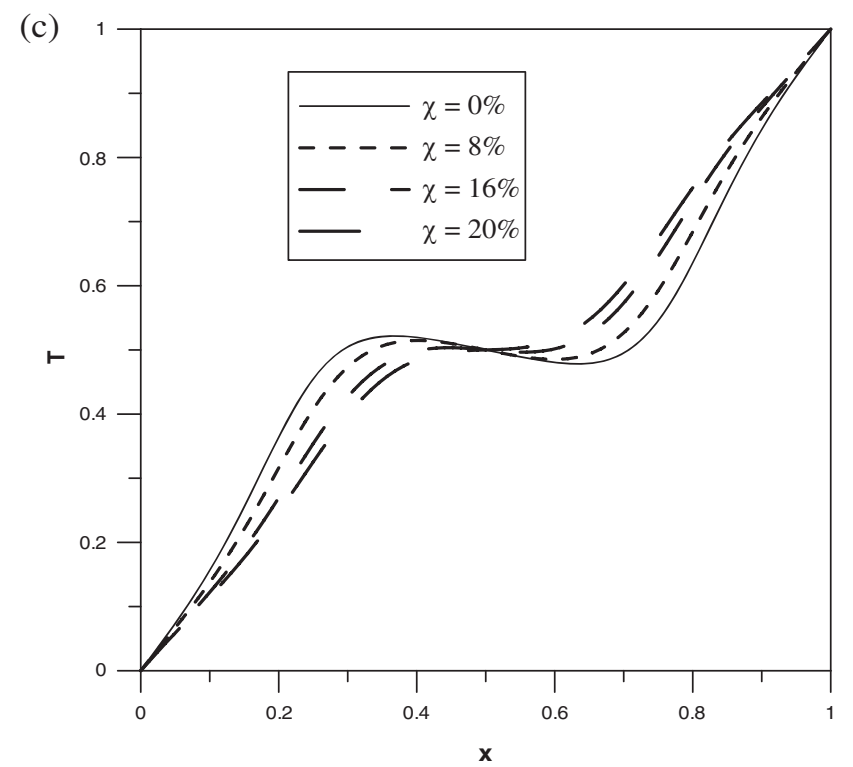

Figure 7: Horizontal mid-plane temperature profile: (a) $\mathrm{Ri}=0.1$; (b) $\mathrm{Ri}=1$; (c) $\mathrm{Ri}=10$ 

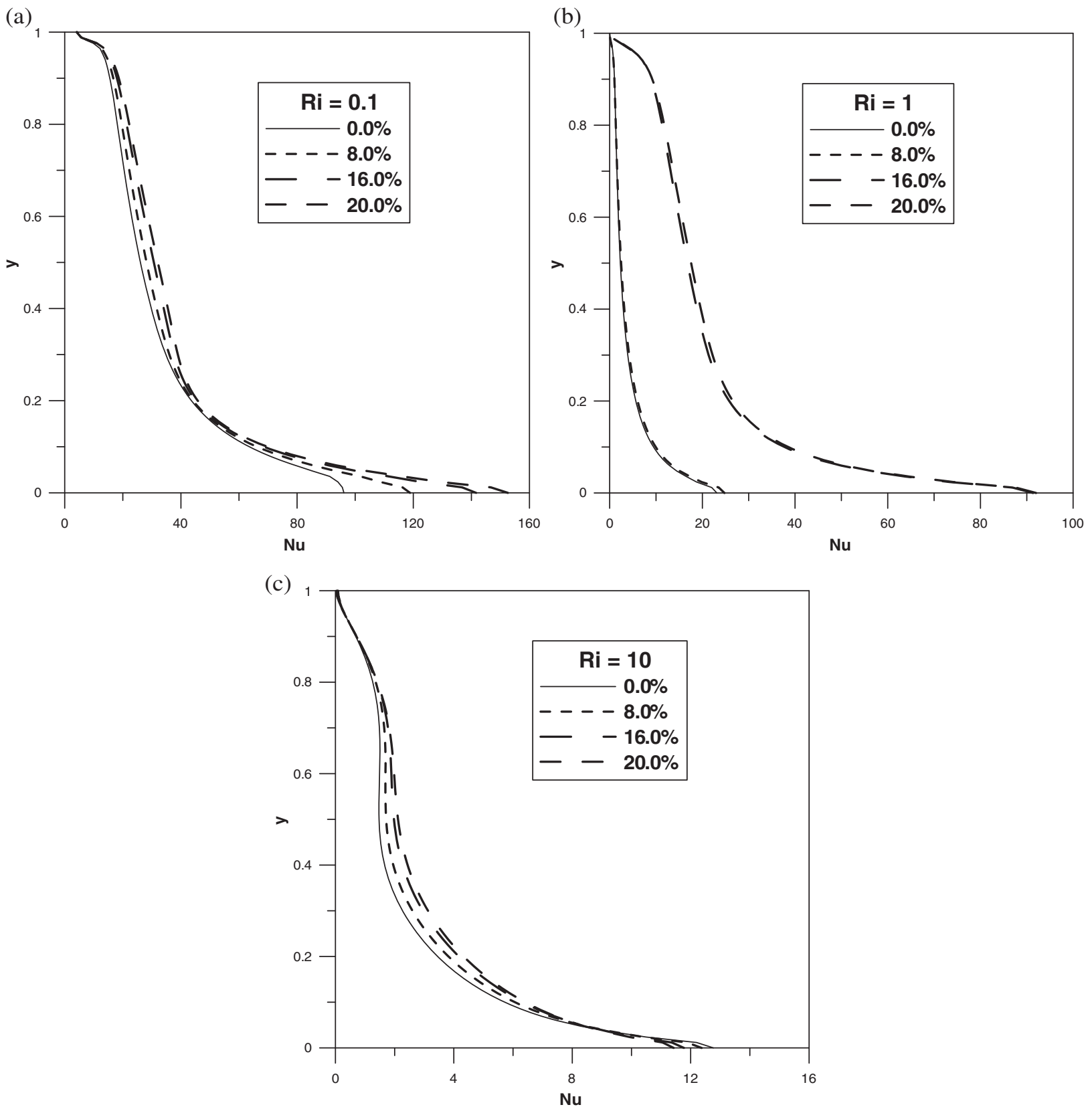

Figure 8: Local Nusselt number at the left wall: (a) $\mathrm{Ri}=0.1$; (b) $\mathrm{Ri}=1$; (c) $\mathrm{Ri}=10$

\subsection{Effect of the Nanoparticles Type}

In this subsection, we shall concentrate our attention on highlighting the effect of the type of nanoparticles on the pattern formation as well as in the heat transfer into the cavity. For that, five types of nanoparticles are taken into consideration such as: $\mathrm{Ag}, \mathrm{Cu}, \mathrm{TiO}_{2}, \mathrm{Fe}_{3} \mathrm{O}_{4}$ and $\mathrm{Al}_{2} \mathrm{O}_{3}$. The obtained results in this framework show that the fluid dynamics is not influenced by changing the type of the nanoparticles in the sense that we obtain the same streamlines and isotherms discussed above for the $\mathrm{Ag}$ nanoparticles. We will not here present these results for avoiding any kind of repetition in the paper. However, the type of nanoparticles affects the heat transfer in the cavity as shown in Fig. 9 where 
evolution of the average Nusselt number versus the volume fraction is displayed for the five types of nanoparticles and for the different values of Richardson. As one can see, we recover the classical observation claiming that the presence of nanoparticles enhances the heat transfer in the cavity in comparison to the pure base fluid independently on whether the natural convection is more or less dominant in comparison to the forced convection. This effect depends on the nature of the nanoparticles and becomes more pronounced in the high values of the volume fraction. Indeed, the parameter $\overline{N u}$ is more important for $\mathrm{Al}_{2} \mathrm{O}_{3}$ than the other nanoparticles when $\mathrm{Ri}=0.1$ since $\overline{N u}_{\mathrm{Al}_{2} \mathrm{O}_{3}}>\overline{\mathrm{Nu}}_{\mathrm{Cu}}>\overline{\mathrm{Nu}}_{\mathrm{Fe}_{3} \mathrm{O}_{4}}>\overline{\mathrm{Nu}}_{\mathrm{TiO}_{2}}>\overline{\mathrm{Nu}}_{\mathrm{Ag}}$. By decreasing the Reynolds number up to $\mathrm{Re}=100$, which correspond to $\mathrm{Ri}=1$, the heat transfer is not influenced by the type of the nanoparticles except for highly concentrated solutions at $\chi>12 \%$ where a slight increase in the average Nusselt number is observed following the same order $\overline{N u}_{\mathrm{Al}_{2} \mathrm{O}_{3}}>\overline{\mathrm{Nu}}_{\mathrm{Cu}}>\overline{\mathrm{Nu}}_{\mathrm{Fe}_{3} \mathrm{O}_{4}}>\overline{\mathrm{Nu}}_{\mathrm{TiO}_{2}}>\overline{\mathrm{Nu}}_{\mathrm{Ag}}$. However, this is not the case when $\mathrm{Ri}=10$ where the parameter $\overline{N u}$ increases nonlinearly with the nanoparticles volume fraction. The effect of the type of the nanoparticles is not similar to the cases $\mathrm{Ri}=0.1$ and $\mathrm{Ri}=1$ where the average Nusselt number is more important for metals nanoparticles, $\mathrm{Cu}$ and $\mathrm{Ag}$, than the oxides ones such as $\overline{N u}_{C u}>\overline{N u}_{A g}>\overline{N u}_{\mathrm{Al}_{2} \mathrm{O}_{3}}>\overline{N u}_{\mathrm{Fe}_{3} \mathrm{O}_{4}}>\overline{N u}_{\mathrm{TiO}_{2}}$.

These features can be explained of the basis of the thermophysical properties of each substance and there implications depending on the mechanism driving the flow. Indeed, when $\mathrm{Ri}=10$ the flow is driven by natural convection and consequently the thermal properties of the nanoparticles especially the thermal conductivity, thermal expansion coefficient and heat capacity play a significant role and mainly contribute in the flow dynamics as well as in the heat transfer in comparison to the hydrodynamical quantities such as the dynamic viscosity and the mixture's density. However, in the case of weak values of Ri the flow is driven mainly by the walls velocities and hence the hydrodynamic quantities are more involved than the thermal ones. In addition, the thermal behavior obtained in this study confirm that the nanofluids heat transfer depends not only on the thermal conductivity of the nanoparticles and the dynamic viscosity of the nanofluid as suggested by several studies in the literature but it depends on a combination of all the thermophysical properties of these substances. To confirm this statement, one can observe from the Tabs.

1 and 2 that

$$
\begin{gathered}
C_{p_{\mathrm{Al}_{2} \mathrm{O}_{3}}}>C_{p_{\mathrm{TiO}_{2}}}>C_{p_{\mathrm{Fe}_{3} \mathrm{O}_{4}}}>C_{p_{\mathrm{Cu}}}>C_{p_{A g}}, \\
\beta_{A g}>\beta_{C u}>\beta_{\mathrm{TiO}_{2}}>\beta_{\mathrm{Al}_{2} \mathrm{O}_{3}}>\beta_{\mathrm{Fe}_{3} \mathrm{O}_{4}} \\
\text { and } k_{\mathrm{Ag}}>k_{\mathrm{Cu}}>k_{\mathrm{Al}_{2} \mathrm{O}_{3}}>k_{\mathrm{Fe}_{3} \mathrm{O}_{4}}>k_{\mathrm{TiO}_{2}}
\end{gathered}
$$

while for the average Nusselt number none of these orders is conserved.

One the other hand, the local Nusselt number at the left cold wall varies versus the vertical coordinate with the same shape meaning that it always decreases versus y. Also, this transfer parameter is not remarkably influenced by the type of the nanoparticles as shown in Fig. 10 at the volume fraction $\chi=20 \%$. In fact, a slight effect is observed at $\mathrm{Ri}=0.1$ especially at the lower part of the wall $0.1<y \leq 0.3$. For instance at $\mathrm{y} \approx 0.12$ the local Nusselt number varies between $N u \approx 58$ for the Ag nanoparticles and $N u \approx 67$ for $\mathrm{Al}_{2} \mathrm{O}_{3}$. At this location we have $N u_{\mathrm{Al}_{2} \mathrm{O}_{3}}>N u_{\mathrm{Fe}_{3} \mathrm{O}_{4}} \approx N u_{\mathrm{TiO}_{2}}>N u_{\mathrm{Cu}}>N u_{A g}$. The same behavior is observed at the upper part of the wall $0.5<y \leq 1$ when $\mathrm{Ri}=10$, see Fig. 10c. Indeed, at $\mathrm{y}=0.7$ the local Nusselt number varies between $N u \approx 1.8$ for the $\mathrm{Fe}_{3} \mathrm{O}_{4}$ and $\mathrm{T}_{\mathrm{i}} \mathrm{O}_{2}$ nanoparticles and $N u \approx 2.2$ for Ag. At this location we have $\mathrm{Nu}_{\mathrm{Ag}}>\mathrm{Nu} u_{\mathrm{Cu}}>\mathrm{Nu}_{\mathrm{Al}_{2} \mathrm{O}_{3}}>\mathrm{Nu}_{\mathrm{Fe}_{3} \mathrm{O}_{4}} \approx N u_{\mathrm{TiO}_{2}}$.

To conclude, the effect of the nature of the nanoparticles on the enhancement of the heat transfer observed in this system cannot be understood on the basis of the present study. However, one can confirm that this is a phenomenon that cannot be solely attributed to the higher thermal properties of the added nanoparticles especially when both natural and forced convection regimes are involved in the system. Consequently, much effort has to be deployed in this direction for a better understanding of the mechanism controlling the heat transfer in systems with nanofluids. 

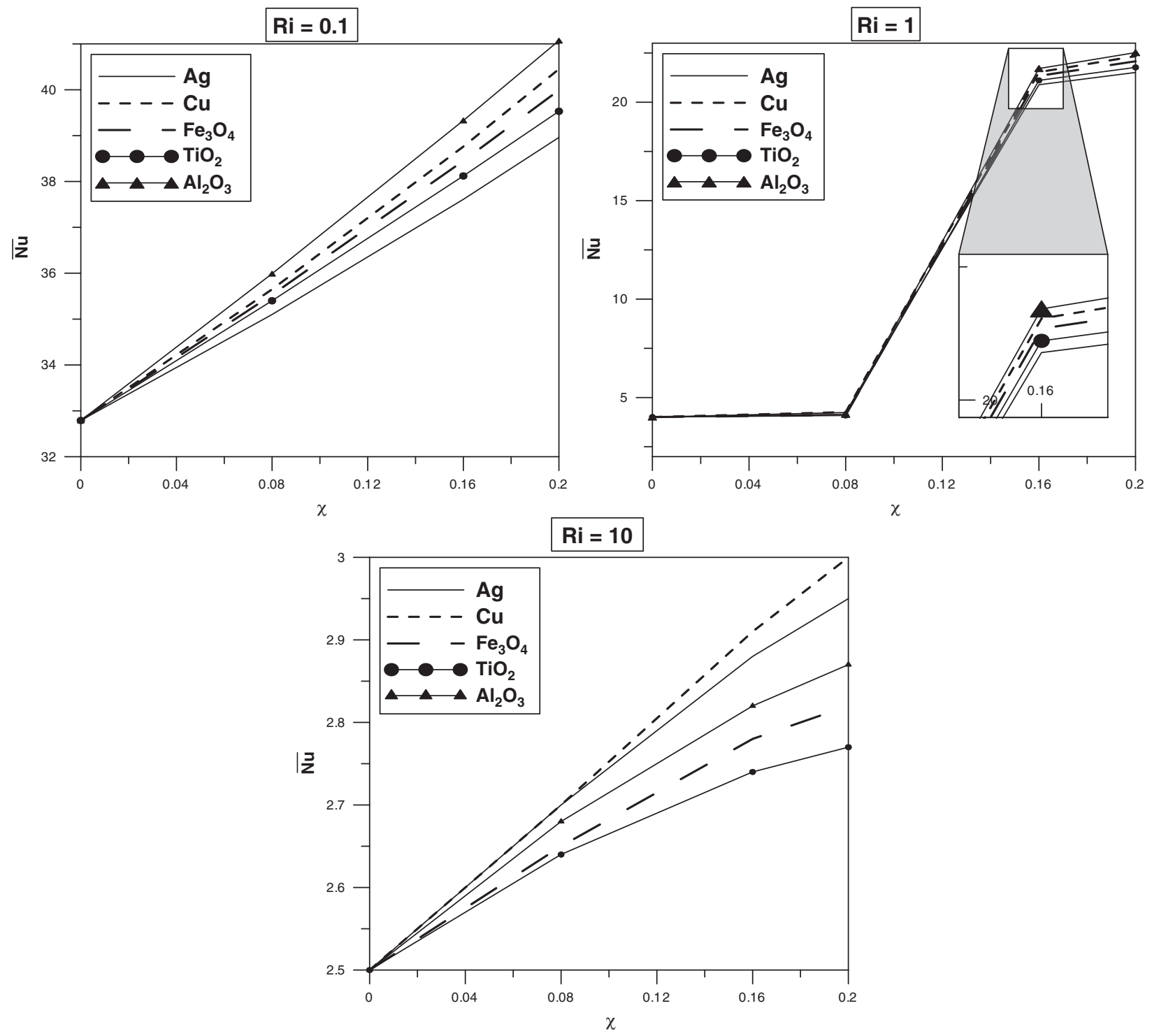

Figure 9: Average Nusselt number versus the volume fraction of the nanoparticles for different values of Richardson number

\subsection{Heat Transfer and Pattern Formation in Ag-Ethylene-Glycol Nanofluid}

Do the heat transfer and the fluid dynamics change when the water is substituted by Ethylene-glycol in this studied configuration? This is the question that we attempt to address in this subsection. For that we consider a nanofluid with Ag-Ethylene-glycol mixture. Streamlines and isotherms related to this solution is depicted in Figs. 11-13 for three different values of Richardson number, $\mathrm{Ri}=0.1,1,10$.

As one can see from Figs. $11 \mathrm{a}-11 \mathrm{c}$ and $11 \mathrm{e}-11 \mathrm{~g}$ ), when $\mathrm{Ri}=0.1$ weak vortices are appeared next to the moving walls whereas next to the horizontal upper/lower walls the streamlines exhibit minimums/maximums that become more accentuated when approaching towards the center of the cavity. The same behavior is observed for the isotherms as depicted in Figs. 11b-11d and $11 \mathrm{f}-11 \mathrm{~h}$. One can also look at the corners of the cavity that show a particular behavior in comparison to the Ag-Water nanofluid. Indeed, the four corners of the cavity are occupied by the nanofluid in contrast to the case of water based 
nanofluid where only the left-upper and right-lower corners that are occupied by the nanofluid. Therefore, the Ethylene-glycol ensures a good distribution of the velocity and the temperature as well as the heat transfer in the cavity in comparison to water. One should also shed light on the formation of a thermal boundary layer in a diagonal direction of the cavity which becomes more pronounced by increasing the nanoparticles volume fraction. This result confirms the increase in the strength of the convection under the effect of the nanoparticles. At $\mathrm{Ri}=1$ where the buoyancy and the shear forces are of the same order of magnitude, different streamlines and isotherms are obtained in comparison to Ag-water nanofluid as displayed in Fig. 12. In this case, the streamlines are characterized by a large central main eddy, occupying the whole of the cavity, breaking into two small ones at the core. This feature remains unchanged by increasing the volume fraction. In addition and regarding the isotherms, thermal boundary layer is spread out over the four walls of the cavity.
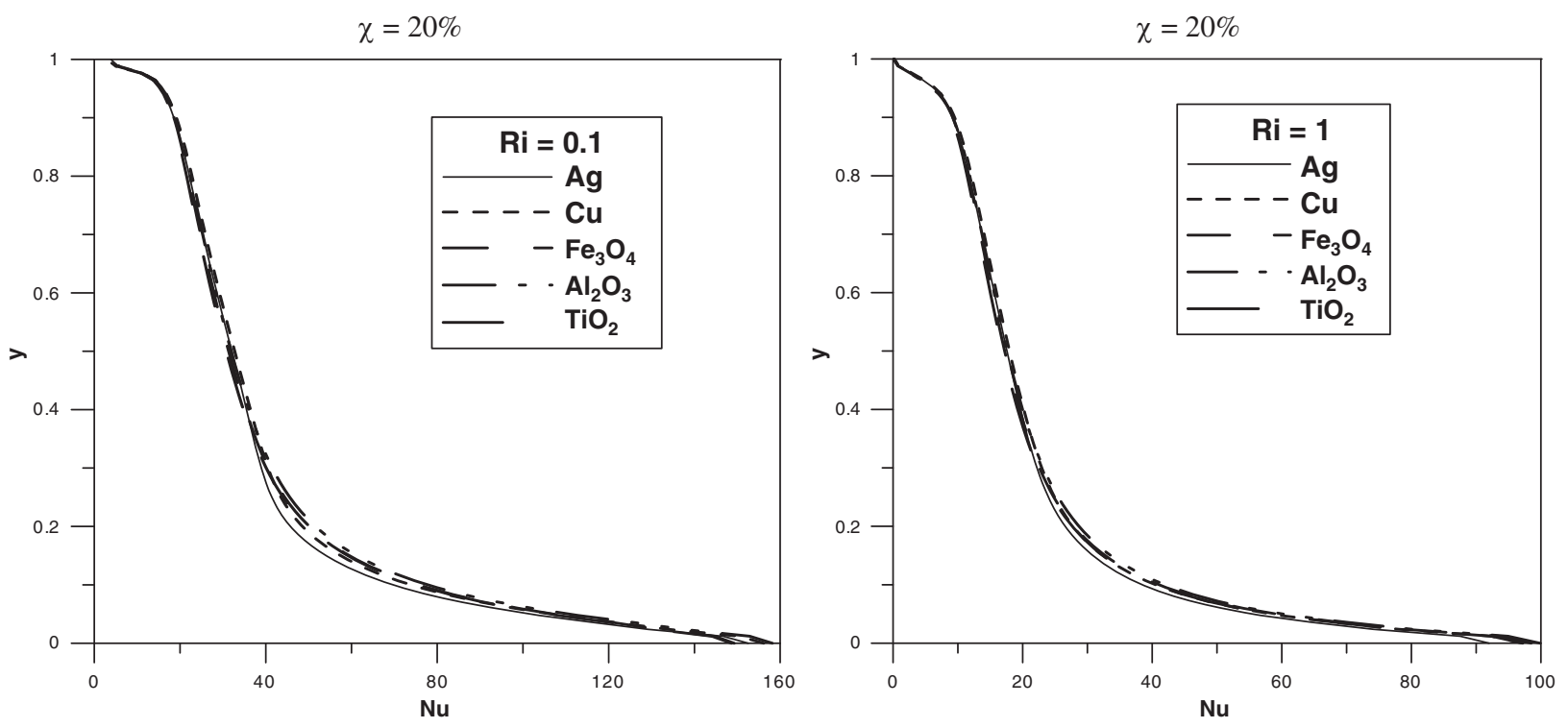

$\chi=20 \%$

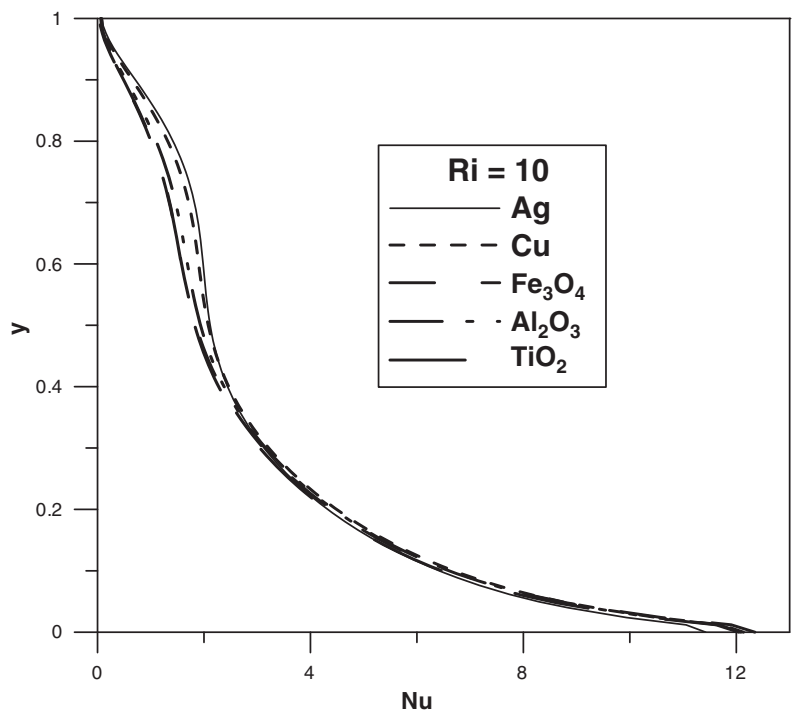

Figure 10: Local Nusselt number at the left wall: (a) $\mathrm{Ri}=0.1$; (b) $\mathrm{Ri}=1$; (c) $\mathrm{Ri}=10$ 
A

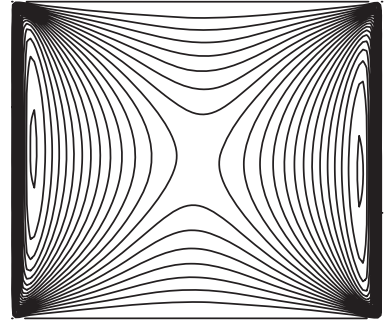

B

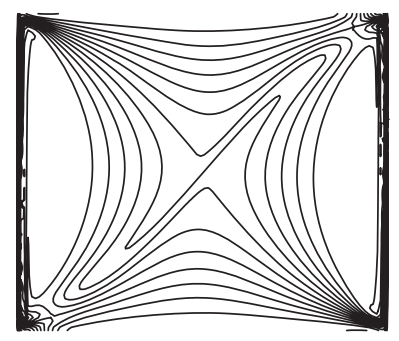

C

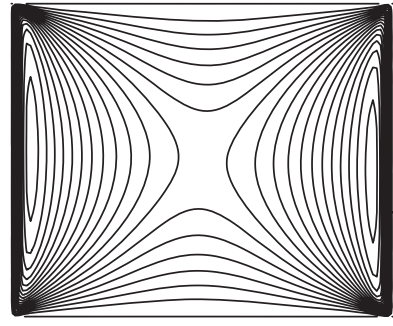

D

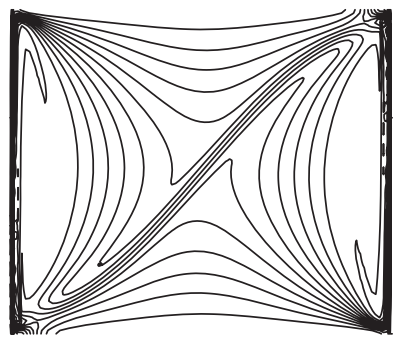

E

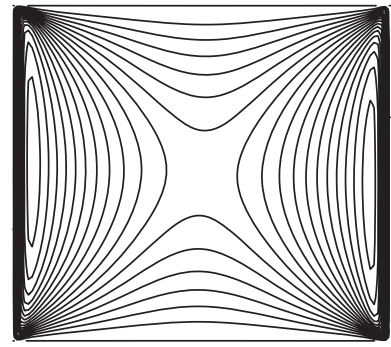

F

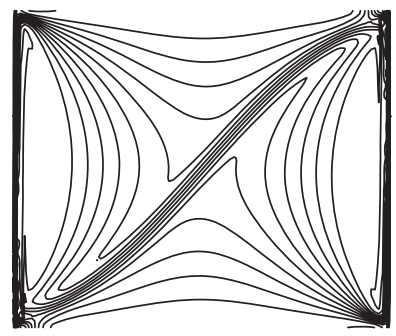

G

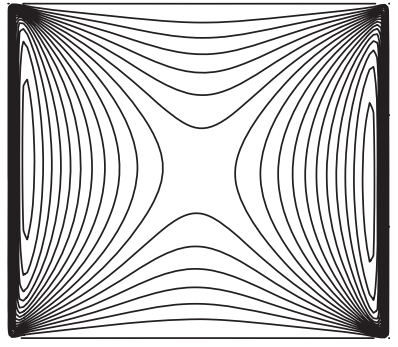

$\mathrm{H}$

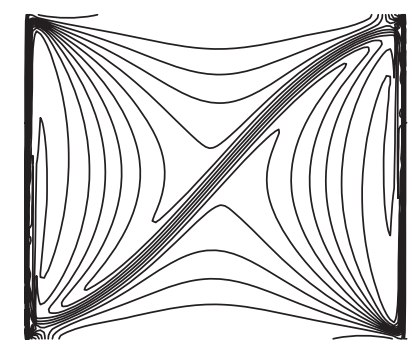

Figure 11: Streamlines (A, C, E, G) and isotherms (B, D, F, H) for Ri $=0.1$ : (A) $\chi=0 \%$; (B) $\chi=0 \%$; (C) $\chi=8 \%$; (D) $\chi=8 \%$; (E) $\chi=16 \%$; (F) $\chi=16 \%$; (G) $\chi=20 \%$; (H) $\chi=20 \%$

A

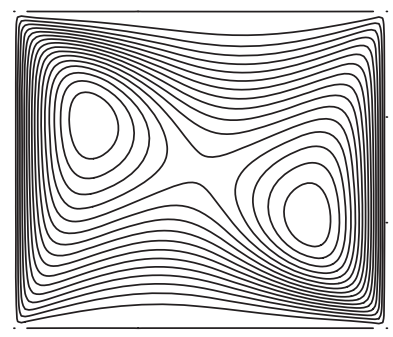

B

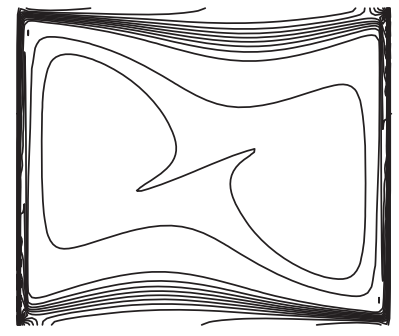

$\mathrm{C}$

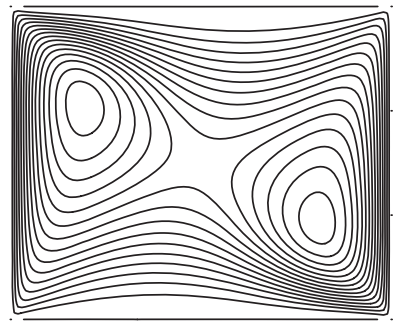

$\mathrm{D}$

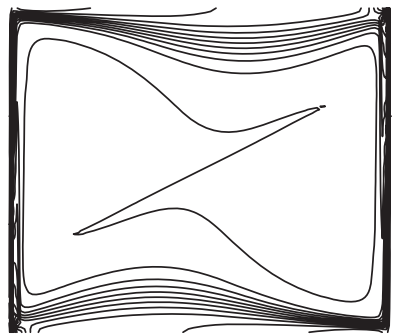

$\mathrm{E}$

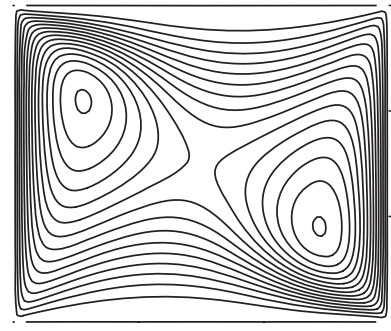

$\mathrm{F}$

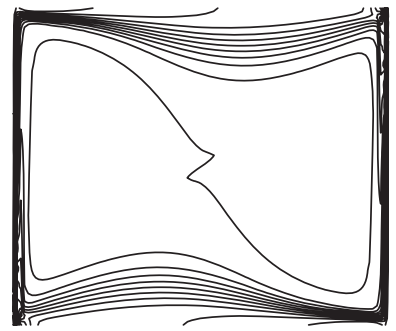

G

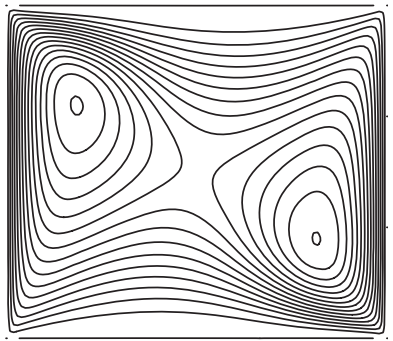

$\mathrm{H}$

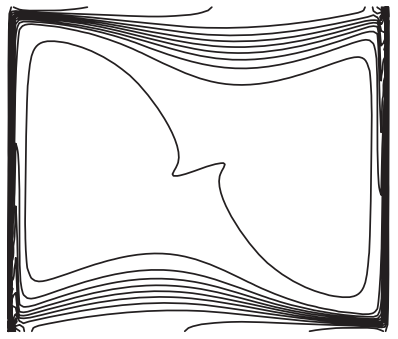

Figure 12: Streamlines (A, C, E, G) and isotherms (B, D, F, H) for Ri $=1$ : (A) $\chi=0 \%$; (B) $\chi=0 \%$; (C) $\chi=8 \%$; (D) $\chi=8 \%$; (E) $\chi=16 \%$; (F) $\chi=16 \%$;(G) $\chi=20 \%$; (H) $\chi=20 \%$

By crossing $\mathrm{Ri}=10$, the flow is characterized by three vortices similarly to the case of Ag-water nanofluid as shown in Fig. 13. The unique difference that should be noticed here is the thickness of the boundary layer formed at the junction of two counter rotating vortices that becomes thinner in comparaison to the case of Ag-water nanofluid. This results reveals the strength of the convection and the heat transfer in the cavity when the used base fluid is the Ethylene-glycol instead of water. The fluid 
velocity in the $x$-direction at the mid plan of the cavity is plotted in Fig. 14 for the three values of the Richardson number, $\mathrm{Ri}=0.1,1,10$.

A

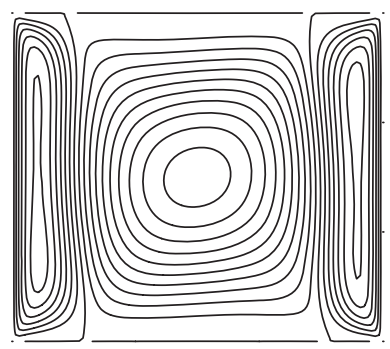

B

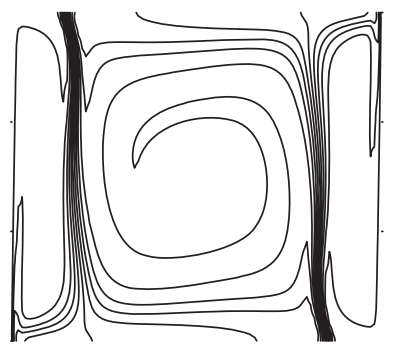

$\mathrm{C}$

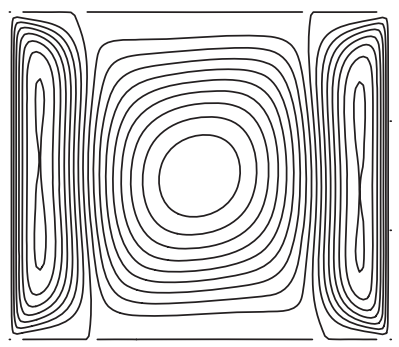

$\mathrm{D}$

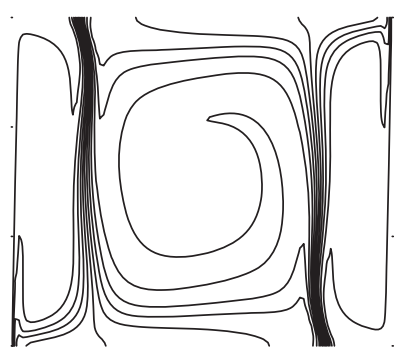

$\mathrm{E}$

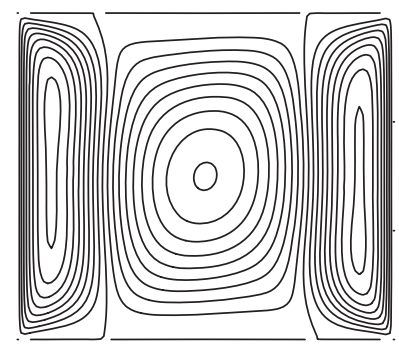

$\mathrm{F}$

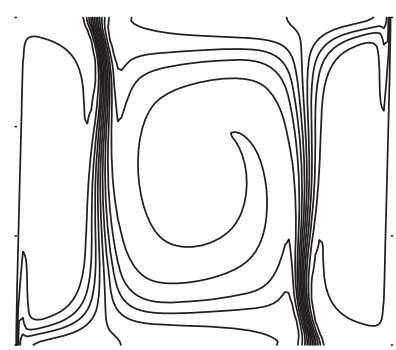

G

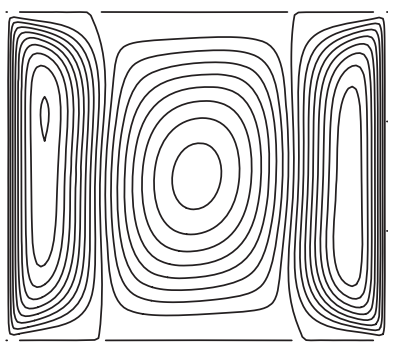

$\mathrm{H}$

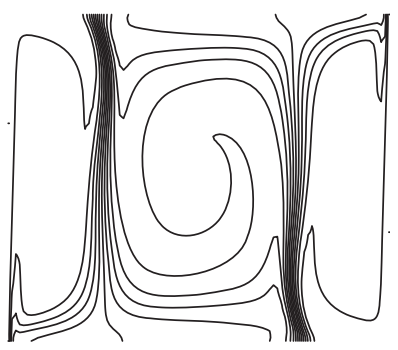

Figure 13: Streamlines (A, C, E, G) and isotherms (B, D, F, H) for Ri $=10$ : (A) $\chi=0 \%$; (B) $\chi=0 \%$; (C) $\chi=8 \%$; (D) $\chi=8 \%$; (E) $\chi=16 \%$; (F) $\chi=16 \%$;

(G) $\chi=20 \%$; (H) $\chi=20 \%$

The shape of this kinematic quantity is symmetric similarly to the Ag-Water nanofluid verifying the Eq. (50). In addition, it is not influenced by the nature of the base fluid in the case $\mathrm{Ri}=0.1$ except a remarkable change in its extrema values, $u_{\max } / u_{\min }$. Indeed, in the case of water based nanofluid the velocity has a maximum of around $u_{\max } \approx 0.65$ whereas in the case of Ethylene-glycol based nanofluid $u_{\max } \approx 0.027$, unlike the case $\mathrm{Ri}=10$ where the velocity extrema are of the same order for both water and Ethylene-glycol. The most important feature that should be highlighted her is the absence of the flow reversal discussed in the case of water based nanofluid especially beyond $\chi=8 \%$. However, a flow reversal is observed by increasing the Richardson number from $\mathrm{Ri}=1$ to $\mathrm{Ri}=10$ independently of the volume fraction of the nanoparticles.

Temperature profile at the horizontal mid-plan related to this configuration is presented in Fig. 15 for different values of Ri. It is apparent that a strong horizontal temperature gradient is observed inside the cavity when $\mathrm{Ri}=0.1$. This feature can be explained on the basis of the nature of the isotherms observed in this case. In addition, this curve exhibits several extrema symmetric with respect to the centre of the cavity obeying the Eq. (51). By increasing the Richardson number to $\mathrm{Ri}=1$, the temperature reaches a constant average value between the temperature of the vertical walls. Near these latter, the nanofluid temperature exhibits two peaks owing to the formation of the thermal boundary layers as described from the isotherms in Fig. 12. Different thermal behavior is observed in the case $\mathrm{Ri}=10$ where the nanofluid temperature varies according to three different regions since the isotherms are composed by three eddies: two lateral eddies and central one. Indeed, near the left cold wall the temperature varies from $T=0$ to $T=0.5$ in the spatial region that corresponds to the left lateral eddy whereas near the right hot wall the temperature varies continuously from $T=0.5$ to $T=1$ in the spatial region that corresponds to the right lateral eddy. Moreover, the temperature reaches an average value $T=0.5$ in a zone where the central eddy is observed. The nanoparticles volume fraction possesses an effect of decreasing and increasing the 
fluid's temperature respectively in the left part of the cavity where $0<x<0.5$ and in the right one where $0.5<x<1$. This effect is observed only when $\mathrm{Ri}=0.1$ and $\mathrm{Ri}=10$ in contrast to the case $\mathrm{Ri}=1$ where no effect of the parameter $\chi$ is observed.

(a)

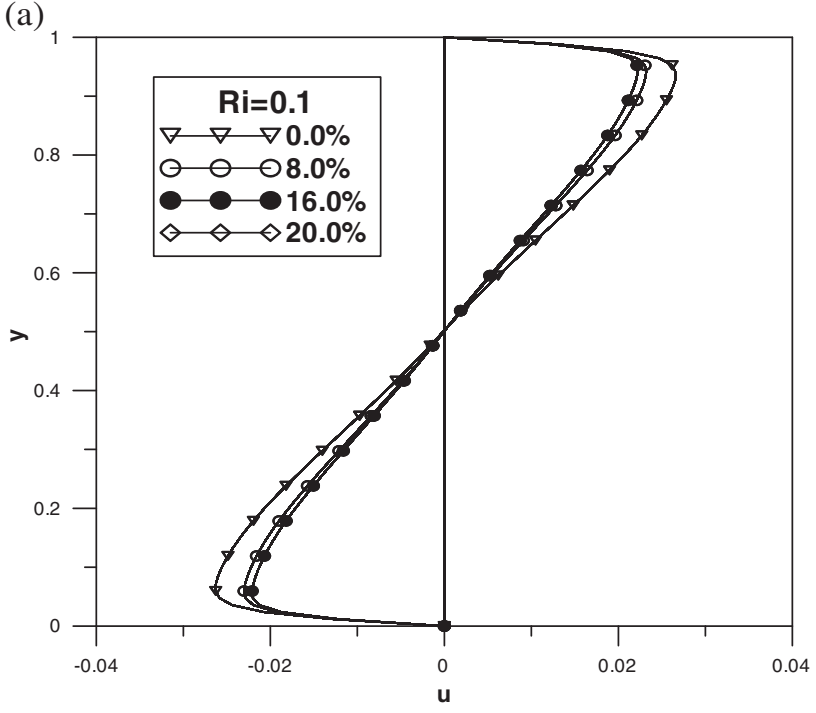

(b)

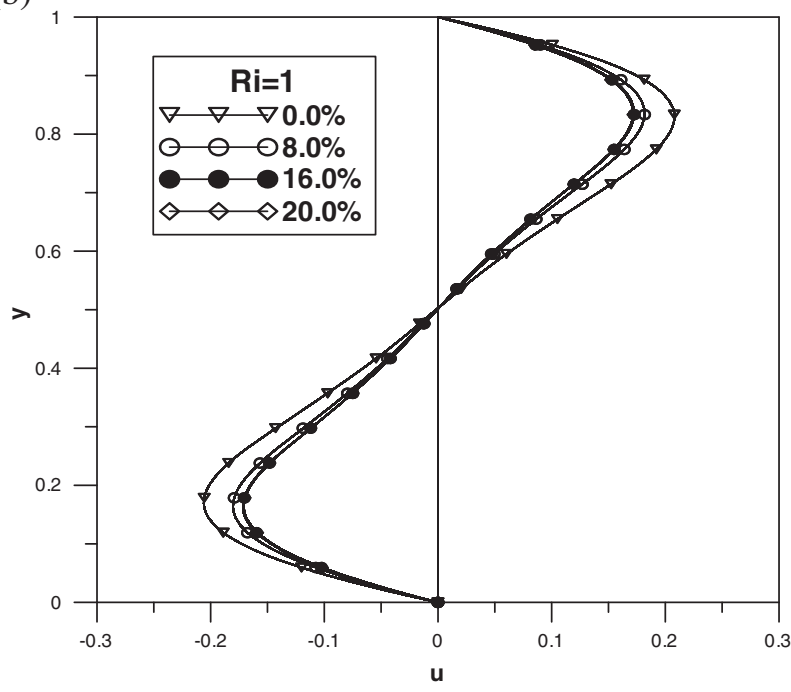

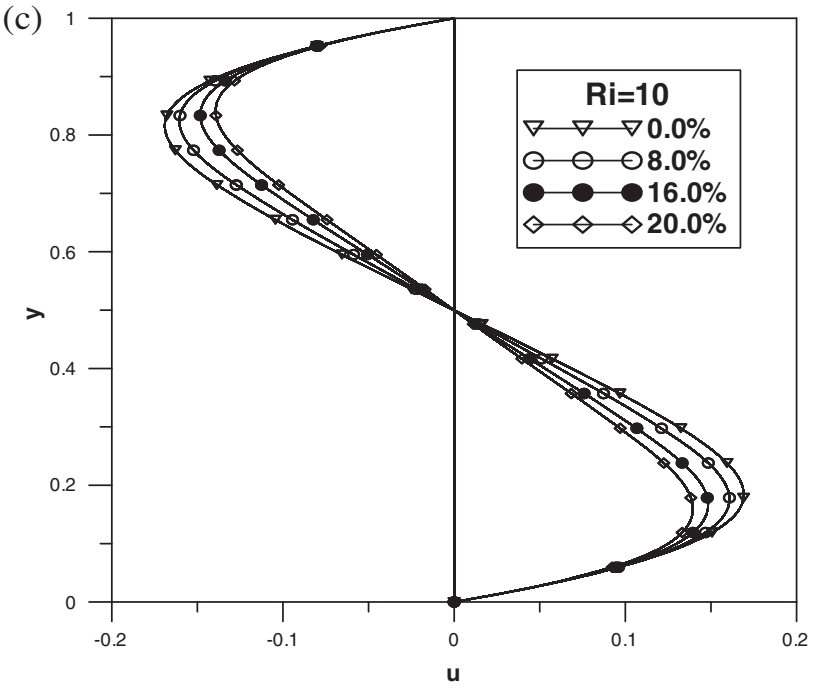

Figure 14: Vertical mid-plane u-velocity: (a) $\mathrm{Ri}=0.1$; (b) $\mathrm{Ri}=1$; (c) $\mathrm{Ri}=10$

\subsection{Effect of the Type of the Base Fluid}

We shall now investigate the heat transfer inside the cavity under the conditions considered in this paper for different type of base fluids. For that, two additional base fluids are added to this study such as Methanol and Kerosene oil beside Water and Ethylene-glycol. Isotherms and streamline related to these fluids (Methanol and Kerosene) will not be presented since they are reminiscent to those obtained in the case of Water. However, drastic differences are obtained in terms of local and average Nusselt numbers. Figs. 16 and 17 show the evolution of the local Nusselt number on the left cold wall in the case of a pure fluid with no-nanoparticles and the case of a maximal nanoparticles volume fraction with $\chi=20 \%$ respectively. As one can see from these figures, when the basis fluid is water the local Nusselt number decreases from the bottom wall to the top having a maximum value at $y=0$. 
(a)

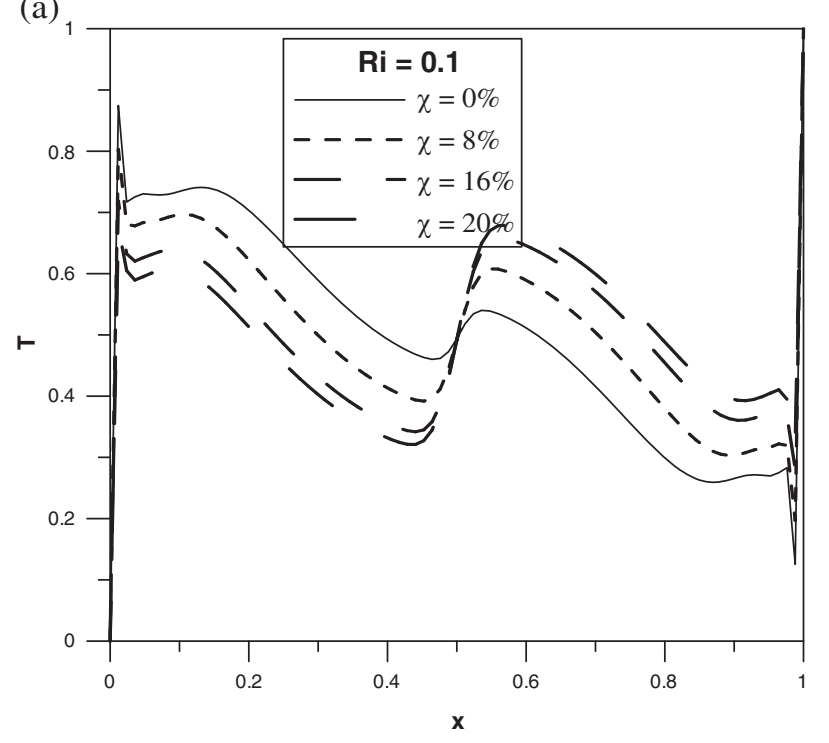

(b)

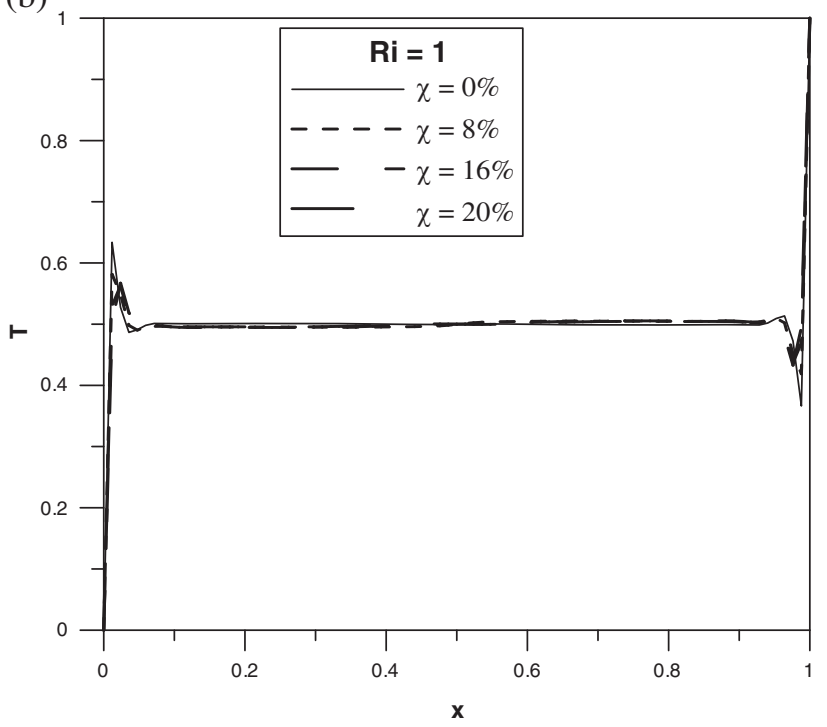

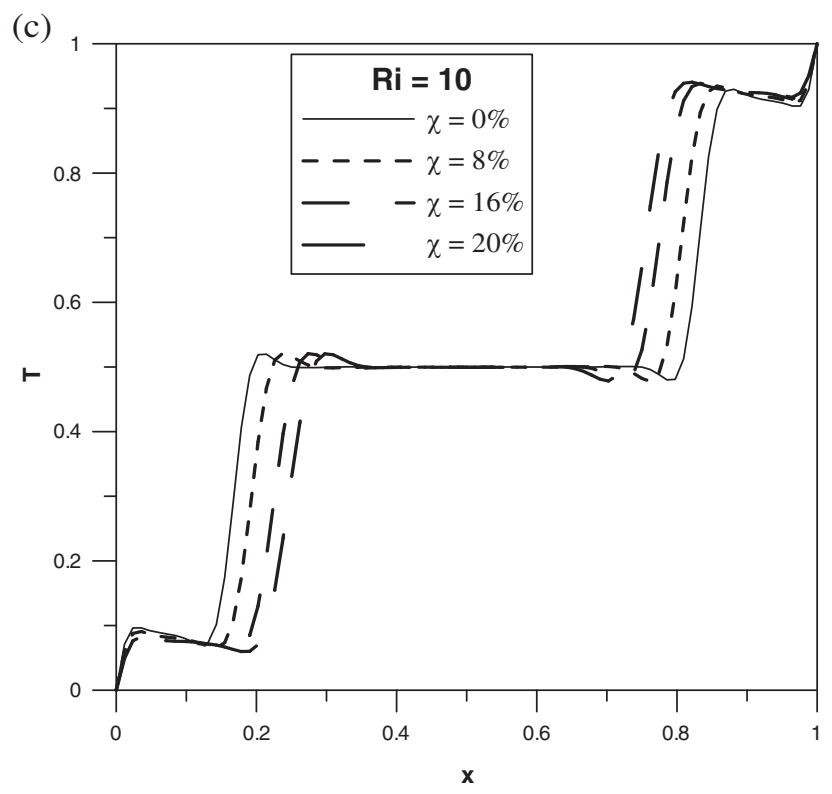

Figure 15: Horizontal mid-plane temperature profile: (a) $\mathrm{Ri}=0.1$; (b) $\mathrm{Ri}=1$; (c) $\mathrm{Ri}=10$

This maximum shifts gradually towards the top of the wall for the others fluids. In addition, it is apparent that the $N u$ number is more important for the Ethylene-glycol base fluid in comparison to the others and one can deduce in general that $N u_{E G}>N u_{\text {Kerosen }}>N u_{\text {Methanol }}>N u_{\text {water }}$. Consequently, the Ethylene-glycol is the most convenient basis fluid ensuring a maximal enhancement of the heat transfer in the cavity. In fact, the difference between the local Nusselt number related to the different fluids varies according to the Richardson number range. For instance, $N u_{\text {Methanol }}, N u_{\text {water }}$ and $N u_{\text {Kerosen }}$ are close to each other at $\mathrm{Ri}=0.1$ in contrast to the cases $\mathrm{Ri}=1$ and $\mathrm{Ri}=10$ where $N u_{\text {Kerosen }}$ becomes much important than $N u_{\text {Methanol }}$ and $N u_{\text {water }}$. It is to be noticed that an increase in the Richardson number leads to a decrease in the local Nusselt number. 

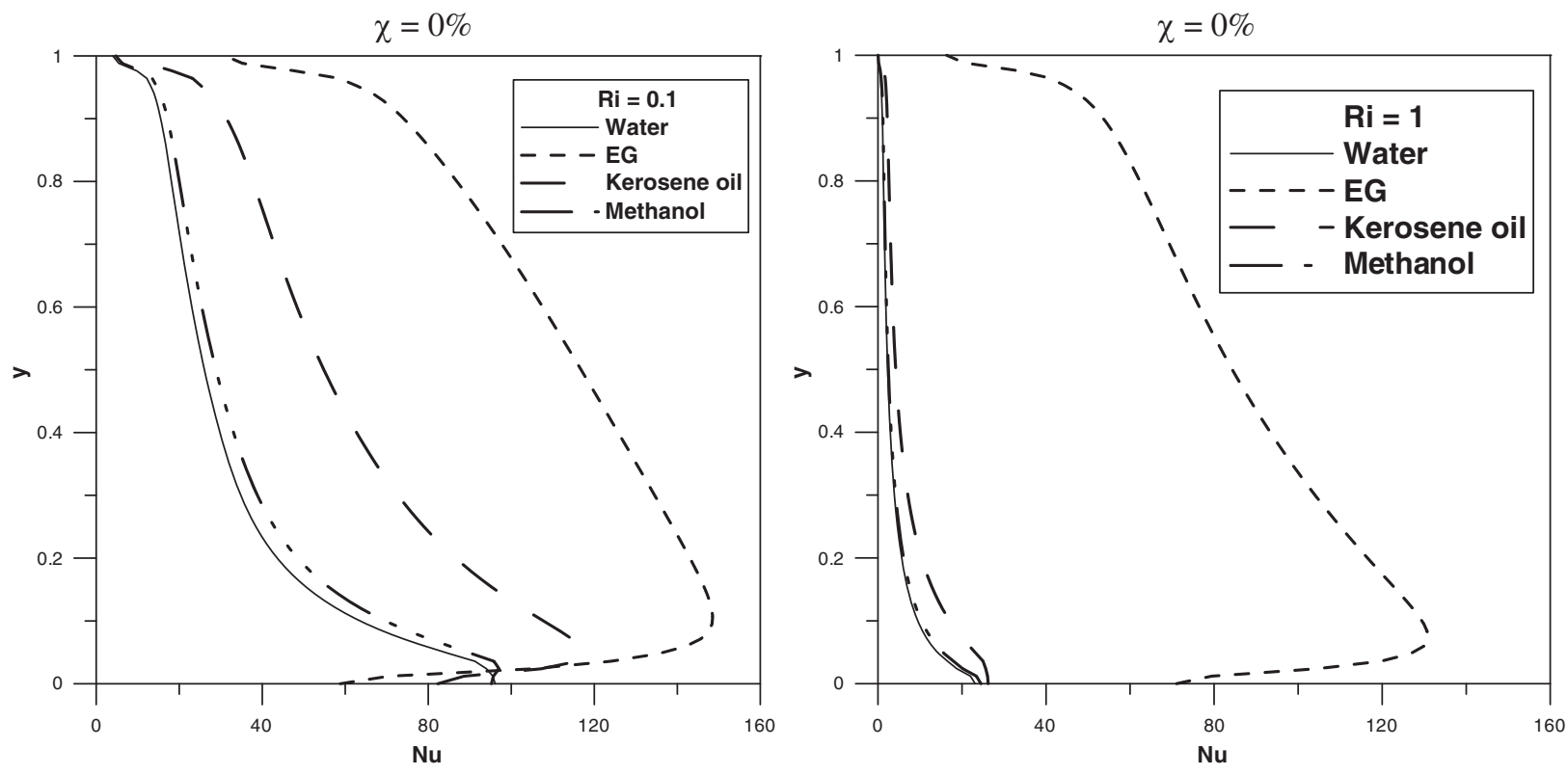

$\chi=0 \%$

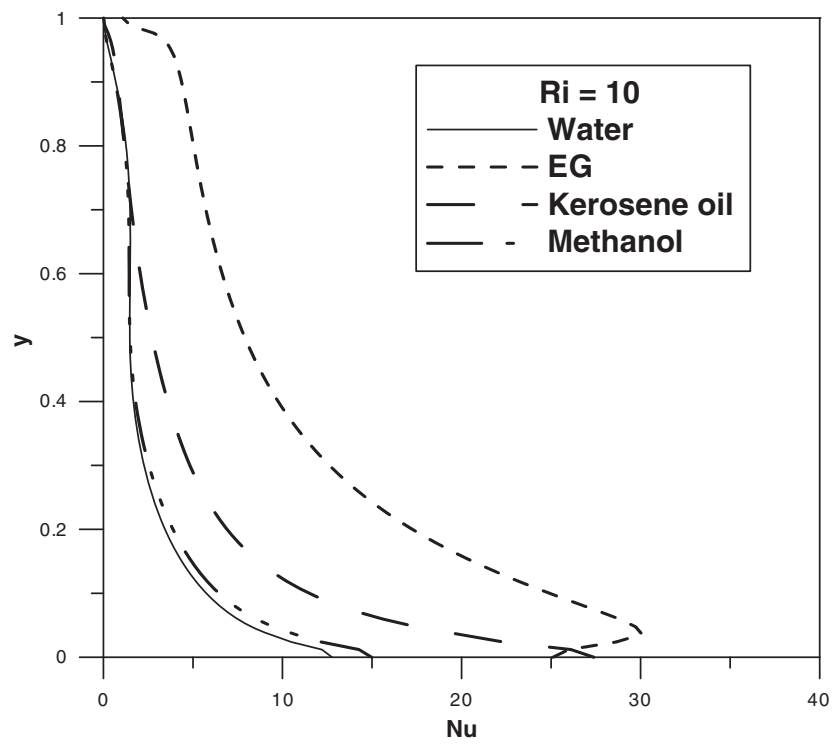

Figure 16: Local Nusselt number at the left wall for different values of Richardson number at $\chi=0 \%$

In order to discern the effect of the Richardson number in the average Nusselt number, $\overline{N u}$, we display in Fig. 18 evolution of this heat transfer quantity as function of Ri for different values of $\chi$. As one can see, $\overline{N u}$ decreases versus Ri more obviously in the Richardson number range $1<\mathrm{Ri}<10$. Under the presence of the nanoparticles the parameter $\overline{N u}$ becomes more important especially in the Richardson number range $0.1<\mathrm{Ri}<1$ for water, Kerosene and Methanol fluids. It is to be noticed that a great change in the concavity of these curves is observed under the effect of increasing the nanoparticles concentration especially for water Kerosene oil and Methanol. This feature can be explained by the fact that the presence of the nanoparticles plays a crucial role in heat transfer enhancement when $R a$ and $R e^{2}$ are of the same order of magnitude which correspond to $\mathrm{Ri}=1$. 

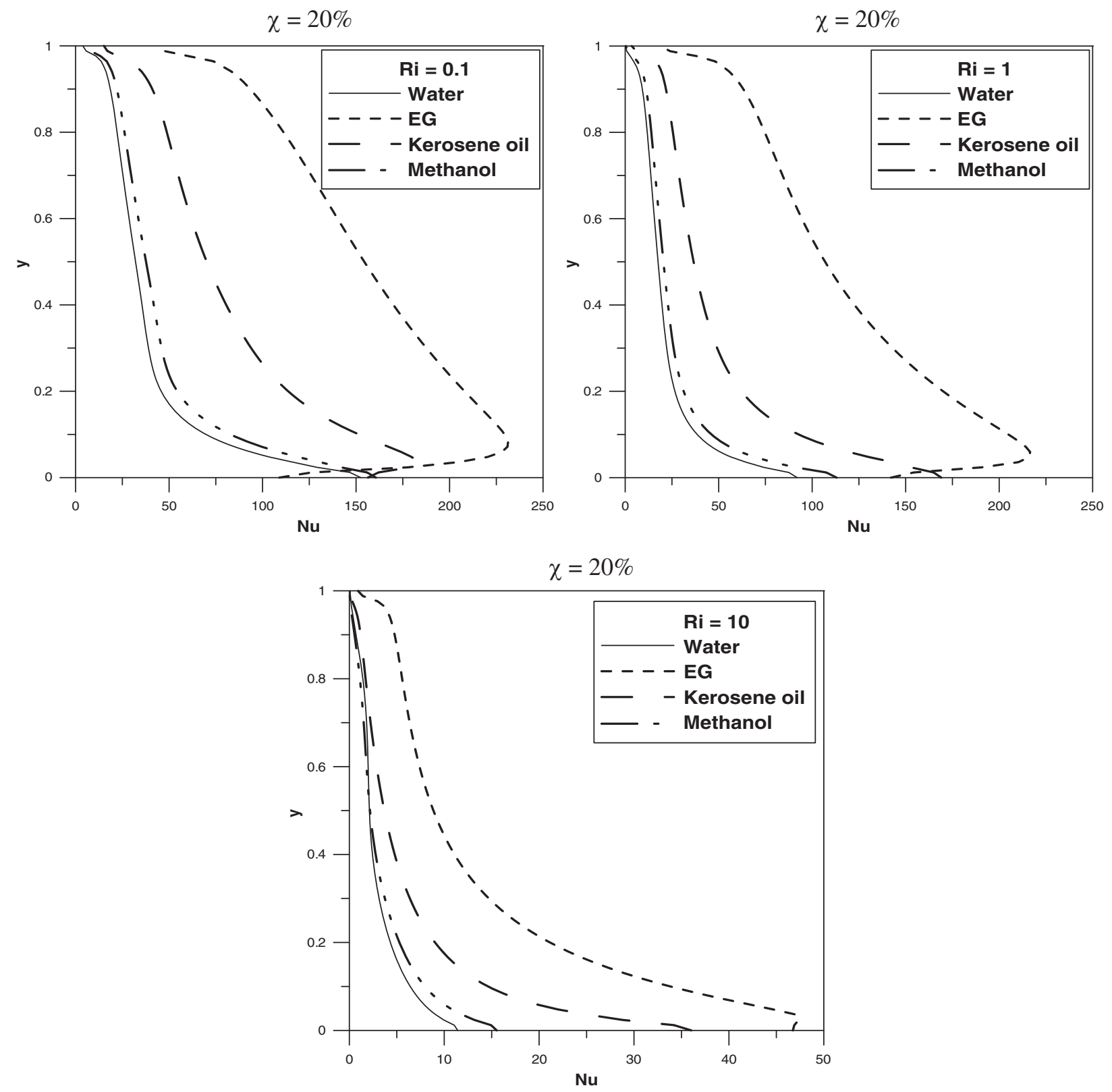

Figure 17: Local Nusselt number at the left wall for different values of Richardson number at $\chi=20 \%$

Indeed, in the neighborhood of this value and in the case of Kerosene oil for example, an increase of the nanoparticles concentration to $\chi=8 \%$ is sufficient to increase the average Nusselt number from approximately 6 at $\chi=0 \%$ to 13.4 . This not the case of Water and Methanol where $\chi=16 \%$ is required to get such jump in the average Nusselt number.

\subsection{Effect of the Nanoparticles Shape and Dynamic Viscosity Law}

In this subsection, we attempt to shed light on the effect of the shape of the nanoparticles on the heat transfer using another thermal conductivity distribution involving cylindrical nanotubes particles given by the following expression: 
$\frac{\kappa_{n f}}{\kappa_{f}}=\frac{\kappa_{s}+\frac{1}{2} \kappa_{f}-\frac{1}{2} \chi\left(\kappa_{f}-\kappa_{s}\right)}{\kappa_{s}+\frac{1}{2} \kappa_{f}+\chi\left(\kappa_{f-} \kappa_{s}\right)}$.
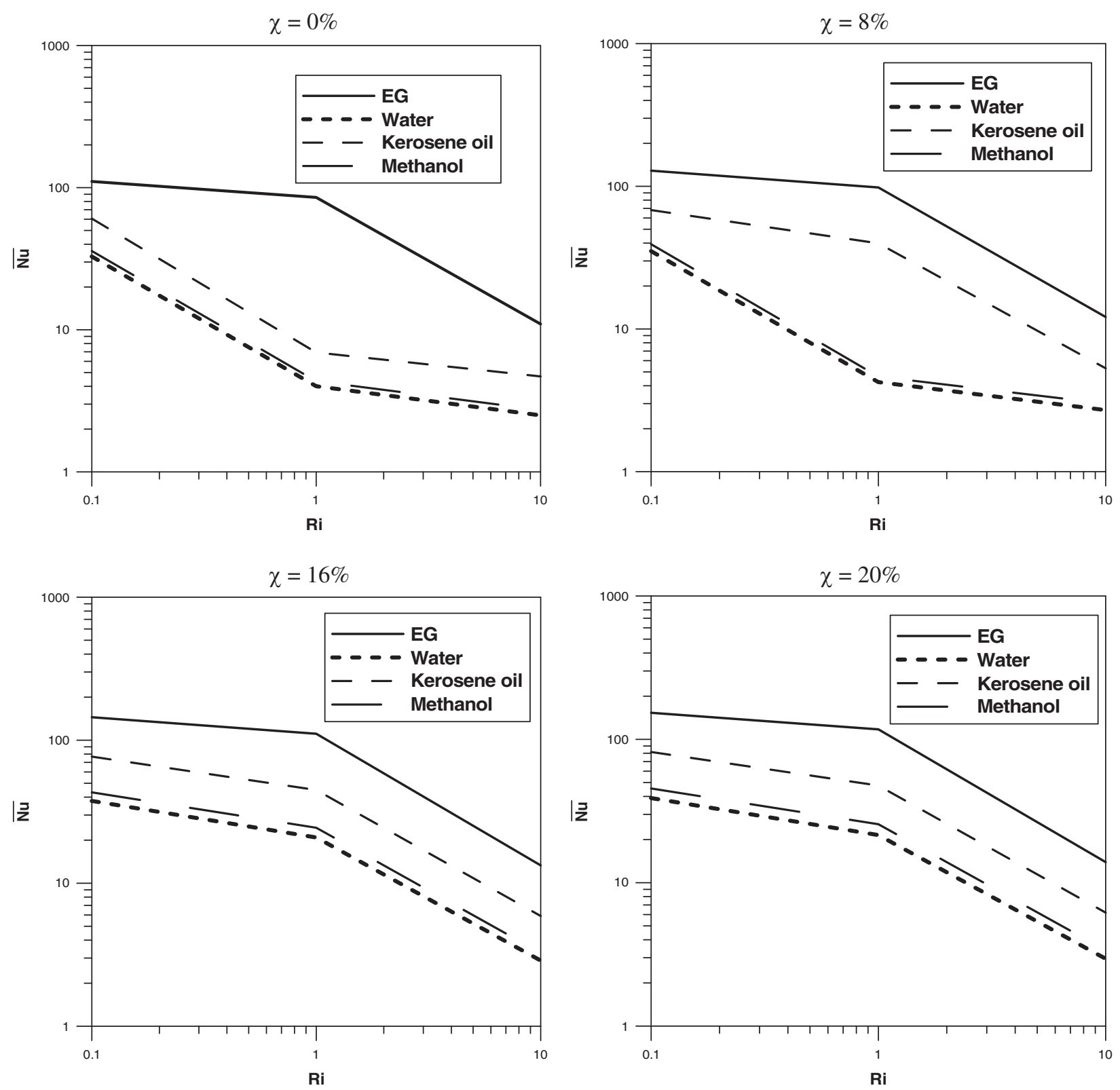

Figure 18: Average Nusselt number versus Richardson number for different volume fraction

In addition, a second dynamic viscosity model, that we term Model II, different to that described by Einstein expression (8) (Model I), is introduced. This model, stemmed from experimental characterizations and used by several authors in the literature [2], is given by 
$\mu_{n f}=\mu_{f}\left(1+7.3 \chi+123 \chi^{2}\right)$.

We consider the case of the Ag-water nanofluid with the volume fraction $\chi=20 \%$ and two types of shape: spherical and cylindrical shapes. It is to be noted that in the framework of the present investigation, the nanoparticles's shape is considered to affect only the dynamic viscosity as well as the thermal conductivity of the nanofluid. Although this approach is widely used in the literature [2] it does not provide a real physical description. However, it is used here to address a picture on how the heat transfer in mixed convection system is influenced by the shape of the nanoparticles.

In Fig. 19, we compare the average Nusselt number related to these shapes using these viscosity models. As one can notice, an enhancement of the heat transfer is observed using the spherical nanoparticles in comparison to the cylindrical ones. Also, a nearly constant decrease in the average Nusselt number $v s$. Ri is noticed using Model II. This is not the case when using Model I where this number, $\overline{N u}$, decreases with a rate of about 16.66 in the range $0<\mathrm{Ri}<1$ and a rate of about 1.75 in the range $1<\mathrm{Ri}<10$. It is also to be noted that at $\mathrm{Ri}=10$, the obtained average Nusselt number using both the spherical nanoparticles with Model I is $\overline{N u}=0.2$ while by the use of the Model II an increase of $\overline{N u}$ is observed where $\overline{N u}=11$.
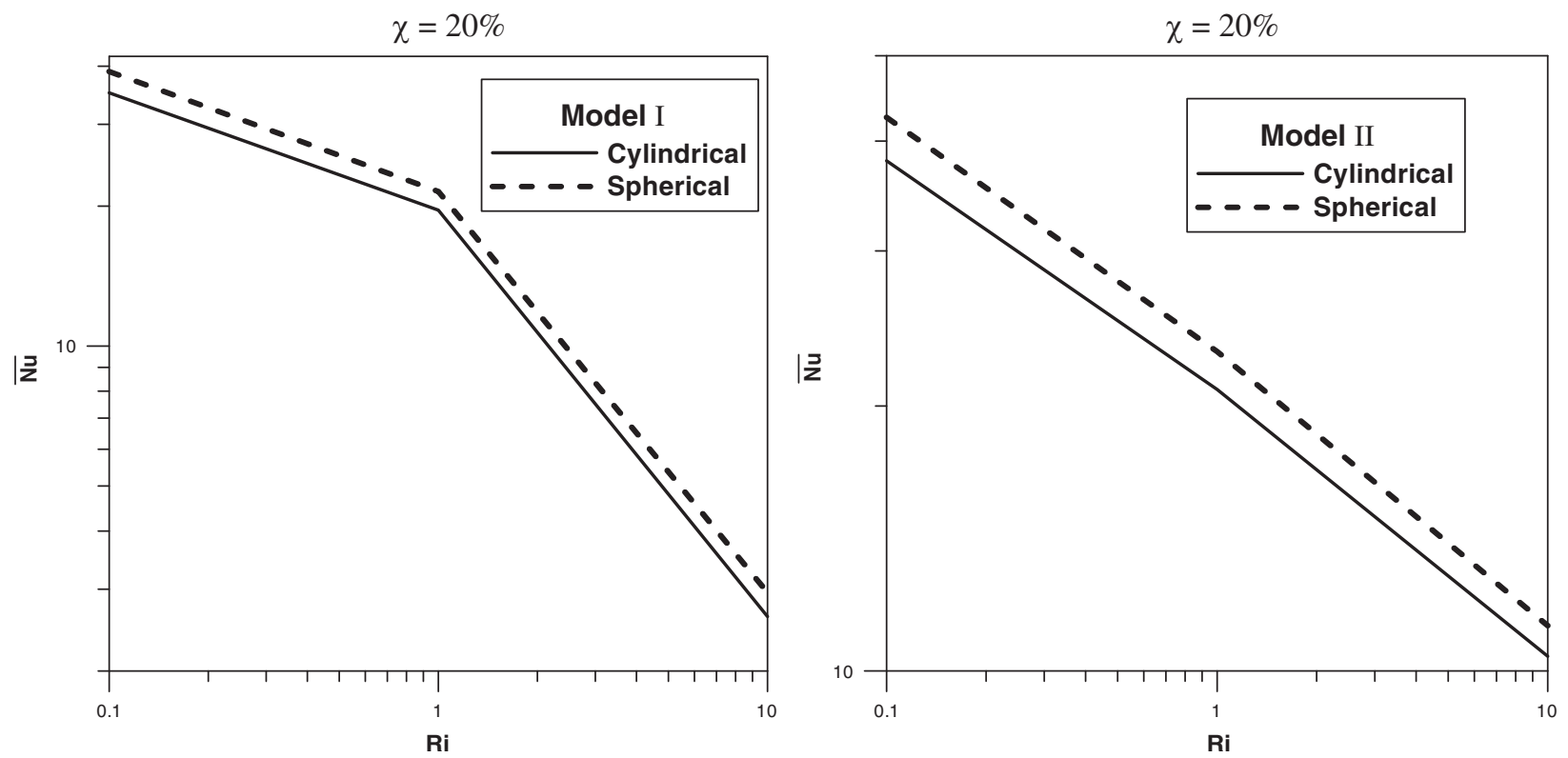

Figure 19: Average Nusselt number versus the Richardson number at $\chi=20 \%$ : (a) Model I and (b) Model II

\section{Conclusions}

In this work, the mixed convection problem in a lid-driven square cavity filled with nanofluid is numerically investigated. The left and right vertical walls move up and down, respectively, and are held at two different temperatures while the horizontal walls are kept insulated. On the basis of the results presented in this paper, following findings may be summarized.

- The type of the nanoparticles does not affect the pattern formation in the system.

- Depending on the Richardson number range, the type of the nanoparticles has a significant role in the heat transfer in terms of both local and average Nusselt number. 
- Different dynamics is observed when the Ethylene-glycol base fluid is used in comparison to water, methanol and kerosene oil.

- Ethylene glycol is the most convenient base fluid, ensuring maximum optimization of heat transfer in the cavity.

- The presence of nanoparticles always increases the heat transfer independently of neither the type of the nanoparticles nor the type of the base fluid.

- Increasing the Richardson number leads to a decrease in the heat transfer for the different mixtures.

- An improvement in heat transfer using spherical nanoparticles compared to cylindrical nanoparticles.

Funding Statement: The author received no specific funding for this study.

Conflicts of Interest: The authors declare that they have no conflicts of interest to report regarding the present study.

\section{References}

1. Tiwari, R. K., Das, M. K. (2007). Heat transfer augmentation in a two-sided lid-driven differentially heated square cavity utilizing nanofluids. International Journal of Heat and Mass Transfer, 50(9-10), 2002-2018. DOI 10.1016/ j.ijheatmasstransfer.2006.09.034.

2. Rana, P., Bhargava, R. (2011). Numerical study of heat transfer enhancement in mixed convection flow along a vertical plate with heat source/sink utilizing nanofluids. Communications in Nonlinear Science and Numerical Simulation, 16(11), 4318-4334. DOI 10.1016/j.cnsns.2011.03.014.

3. Zeghbid, I., Bessaïh, R. (2017). Mixed convection in a lid-driven square cavity with heat sources using nanofluids. Fluid Dynamics \& Materials Processing, 13(4), 251-273.

4. Bensouici, F., Boudebous, S. (2017). Mixed convection of nanofluids inside a lid-driven cavity heated by a central square heat source. Fluid Dynamics \& Materials Processing, 13(3), 189-212.

5. Slama, S., Kahalerras, H., Fersadou, B. (2017). Mixed convection of a nanofluid in a vertical anisotropic porous channel with heated/cooled walls. Fluid Dynamics \& Materials Processing, 13(3), 155-172.

6. Zaraki, A., Ghalambaz, M., Chamkha, A. J., Ghalambaz, M., de Rossi, D. (2015). Theoretical analysis of natural convection boundary layer heat and mass transfer of nanofluids: Effects of size, shape and type of nanoparticles, type of base fluid and working temperature. Advanced Powder Technology, 26(3), 935-946. DOI 10.1016/j. apt.2015.03.012.

7. Raza, J., Farooq, M., Mebarek-Oudina, F., Mahanthesh, B. (2019). Multiple slip effects on MHD non-Newtonian nanofluid flow over a nonlinear permeable elongated sheet: Numerical and statistical analysis. Multidiscipline Modeling in Materials and Structures, 15(5), 913-931. DOI 10.1108/MMMS-11-2018-0190.

8. Levin, M., Miller, M. (1981). Maxwell's "treatise on electricity and magnetism." Uspekhi Fizicheskikh Nauk, 135(11), 425-440. DOI 10.3367/UFNr.0135.198111d.0425.

9. Peaceman, D. W., Rachford, J., Henry, H. (1955). The numerical solution of parabolic and elliptic differential equations. Journal of the Society for Industrial and Applied Mathematics, 3(1), 28-41. DOI 10.1137/0103003.

10. Erturk, E., Gökçöl, C. (2006). Fourth-order compact formulation of Navier-Stokes equations and driven cavity flow at high Reynolds numbers. International Journal for Numerical Methods in Fluids, 50(4), 421-436. DOI 10.1002/fld.1061.

11. Störtkuhl, T., Zenger, C., Zimmer, S. (1994). An asymptotic solution for the singularity at the angular point of the lid driven cavity. International Journal of Numerical Methods for Heat \& Fluid Flow, 4(1), 47-59. DOI 10.1108/ EUM0000000004030.

12. de Vahl Davis, G. (1983). Natural convection of air in a square cavity: A bench mark numerical solution. International Journal for Numerical Methods In Fluids, 3(3), 249-264. DOI 10.1002/fld.1650030305.

13. Markatos, N. C., Pericleous, K. (1984). Laminar and turbulent natural convection in an enclosed cavity. International Journal of Heat and Mass Transfer, 27(5), 755-772. DOI 10.1016/0017-9310(84)90145-5. 
14. Hadjisophocleous, G., Sousa, A., Venart, J. (1988). Prediction of transient natural convection in enclosures of arbitrary geometry using a nonorthogonal numerical model. Numerical Heat Transfer, Part A Applications, 13, 373-392.

15. El Bouihi, I., Sehaqui, R. (2012). Numerical study of natural convection in a two-dimensional enclosure with a sinusoidal boundary thermal condition utilizing nanofluid. Engineering, 4(08), 445-452. DOI 10.4236/ eng.2012.48058.

16. Khanafer, K., Vafai, K., Lightstone, M. (2003). Buoyancy-driven heat transfer enhancement in a two-dimensional enclosure utilizing nanofluids. International Journal of Heat and Mass Transfer, 46(19), 3639-3653. DOI 10.1016/S0017-9310(03)00156-X.

17. Barakos, G., Mitsoulis, E., Assimacopoulos, D. (1994). Natural convection flow in a square cavity revisited: Laminar and turbulent models with wall functions. International Journal for Numerical Methods in Fluids, 18(7), 695-719. DOI 10.1002/fld.1650180705.

18. Fusegi, T., Hyun, J., Kuwahara, K., Farouk, B. (1991). A numerical study of three-dimensional natural convection in a differentially heated cubical enclosure. International Journal of Heat and Mass Transfer, 34(6), $1543-1557$. DOI 10.1016/0017-9310(91)90295-P.

19. Goodarzi, M., Safaei, M., Karimipour, A., Hooman, K., Dahari, M. et al. (2014). Comparison of the finite volume and lattice Boltzmann methods for solving natural convection heat transfer problems inside cavities and enclosures. Abstract and Applied Analysis, 2014, 15. DOI 10.1155/2014/762184.

20. Sharif, M. (2007). Laminar mixed convection in shallow inclined driven cavities with hot moving lid on top and cooled from bottom. Applied Thermal Engineering, 27(5-6), 1036-1042. DOI 10.1016/j.applthermaleng.2006.07.035.

21. Waheed, M. (2009). Mixed convective heat transfer in rectangular enclosures driven by a continuously moving horizontal plate. International Journal of Heat and Mass Transfer, 52(21-22), 5055-5063. DOI 10.1016/j. ijheatmasstransfer.2009.05.011.

22. Khanafer, K. M., Chamkha, A. J. (1999). Mixed convection flow in a lid-driven enclosure filled with a fluidsaturated porous medium. International Journal of Heat and Mass Transfer, 42(13), 2465-2481. DOI 10.1016/ S0017-9310(98)00227-0.

23. Iwatsu, R., Hyun, J. M., Kuwahara, K. (1993). Mixed convection in a driven cavity with a stable vertical temperature gradient. International Journal of Heat and Mass Transfer, 36(6), 1601-1608. DOI 10.1016/ S0017-9310(05)80069-9.

24. Cheng, T. (2011). Characteristics of mixed convection heat transfer in a lid-driven square cavity with various Richardson and Prandtl numbers. International Journal of Thermal Sciences, 50(2), 197-205. DOI 10.1016/j. ijthermalsci.2010.09.012.

25. Riahi, M., Aniss, S., Ouazzani Touhami, M. (2019). Families of reversing and non-reversing Taylor vortex flows between two co-oscillating cylinders with different amplitudes. Physics of Fluids, 31(1), 014101. DOI 10.1063/ 1.5064656.

26. Riahi, M., Aniss, S., Ouazzani Touhami, M., Skali Lami, S. (2016). Centrifugal instability of pulsed TaylorCouette flow in a Maxwell fluid. European Physical Journal E, 39(8), 82. DOI 10.1140/epje/i2016-16082-9.

27. Riahi, M., Aniss, S., Ouazzani Touhami, M., Skali Lami, S. (2015). Pulsed Taylor-Couette flow in a viscoelastic fluid under inner cylinder modulation. European Physical Journal Plus, 130(12), 253. DOI 10.1140/epjp/i201515253-7.

28. Avila, M., Belisle, M. J., Lopez, J. M., Marques, F., Saric, W. S. (2008). Mode competition in modulated TaylorCouette flow. Journal of Fluid Mechanics, 601, 381-406. DOI 10.1017/S0022112008000748. 